\title{
The decimation process in random $k$-SAT
}

\author{
Amin Coja-Oghlan and Angelica Y. Pachon-Pinzon* \\ University of Warwick, Mathematics and Computer Science, \\ Zeeman building, Coventry CV4 7AL, UK \\ $\{a \cdot c o j a-o g h l a n, a \cdot y \cdot p a c h o n-p i n z o n\} @ w a r w i c k \cdot a c \cdot u k$
}

October 11, 2018

\begin{abstract}
Let $\boldsymbol{\Phi}$ be a uniformly distributed random $k$-SAT formula with $n$ variables and $m$ clauses. Non-rigorous statistical mechanics ideas have inspired a message passing algorithm called Belief propagation guided decimation for finding satisfying assignments of $\boldsymbol{\Phi}$. This algorithm can be viewed as an attempt at implementing a certain thought experiment that we call the decimation process. In this paper we identify a variety of phase transitions in the decimation process and link these phase transitions to the performance of the algorithm.

Key words: random structures, phase transitions, $k$-SAT, Belief Propagation.
\end{abstract}

\section{Introduction}

Let $k \geq 3$ and $n>1$ be integers, let $r>0$ be a real, and set $m=\lceil r n\rceil$. Let $\boldsymbol{\Phi}=\boldsymbol{\Phi}_{k}(n, m)$ be a propositional formula obtained by choosing a set of $m$ clauses of length $k$ over the variables $V=\left\{x_{1}, \ldots, x_{n}\right\}$ uniformly at random. For $k, r$ fixed we say that $\boldsymbol{\Phi}$ has some property $\mathcal{P}$ with high probability ('w.h.p.') if $\lim _{n \rightarrow \infty} \mathrm{P}[\boldsymbol{\Phi} \in \mathcal{P}]=$ 1.

The interest in random $k$-SAT originates from the experimental observation that for certain densities $r$ the random formula $\Phi$ is satisfiable w.h.p. while a large class of algorithms, including and particularly the workhorses of practical SAT solving such as sophisticated DPLL-based solvers, fail to find a satisfying assignment efficiently [17]. Over the past decade, a fundamentally new class of algorithms has been proposed on the basis of ideas from statistical physics [6, 16]. Experiments performed for $k=3,4,5$ indicate that these new 'message passing algorithms', namely Belief Propagation guided decimation and Survey Propagation guided decimation ('BP/SP decimation'), excel on random $k$-SAT instances [13]. Indeed, the experiments indicate that BP/SP decimation find satisfying assignments for $r$ close to the threshold where $\boldsymbol{\Phi}$ becomes unsatisfiable w.h.p. Generally, SP decimation is deemed conceptually superior to BP decimation.

For example, in the case $k=4$ the threshold for the existence of satisfying assignments is conjectured to be $m / n \sim r_{4} \approx 9.93$ [15]. According to experiments from [13], SP decimation finds satisfying assignments for densities up to $r=9.73$. Experiments from [19] suggest that the "vanilla" version of BP decimation succeeds up to $r=9.05$. Another version of BP decimation (with a different decimation strategy from [6]) succeeds up to $r=9.24$, again according to experimental data from [13]. By comparison, the currently best rigorously analyzed algorithm is efficient up to $r=5.54$ [10], while zChaff, a prominent practical SAT solver, becomes ineffective beyond $r=5.35[13]$.

Since random $k$-SAT instances have widely been deemed extremely challenging benchmarks, the stellar experimental performance of the physicists' message passing algorithms has stirred considerable excitement. However, the statistical mechanics ideas that BP/SP decimation are based on are highly non-rigorous, and thus a rigorous analysis of these message passing algorithms is an important but challenging open problem. A first step was made in [8], where it was shown that BP decimation does not outperform far simpler combinatorial algorithms for sufficiently large clause lengths $k$. More precisely, the main result of [8] is that there is a constant $\rho_{0}>0$ (independent of $k$ ) such that the 'vanilla' version of BP decimation fails to find satisfying assignments w.h.p. if $r>\rho_{0} 2^{k} / k$. By comparison, non-constructive arguments show that w.h.p. $\Phi$ is satisfiable if $r<r_{k}=2^{k} \ln 2-k$, and unsatisfiable if $r>2^{k} \ln 2$ [3, 4]. This means that for $k \gg \rho_{0}$ sufficiently large, BP decimation fails to find satisfying assignments w.h.p. already for densities a factor of (almost) $k$ below the threshold for satisfiability.

*Supported by EPSRC grant EP/G039070/2 and DIMAP. 
Experiment 2.1 ('decimation process') Input: A satisfiable $k$-CNF $\Phi$.

Result: A satisfying assignment $\sigma: V \rightarrow\{0,1\}$ (with $0 / 1$ representing 'false'/"true').

0. $\quad$ Let $\Phi_{0}=\Phi$.

1. For $t=1, \ldots, n$ do

2. Compute the fraction $M_{x_{t}}\left(\Phi_{t-1}\right)$ of all satisfying assignments of $\Phi_{t-1}$ in which the variable $x_{t}$ takes the value 1 .

3. Assign $\sigma\left(x_{t}\right)=1$ with probability $M_{x_{t}}\left(\Phi_{t-1}\right)$, and let $\sigma\left(x_{t}\right)=0$ otherwise.

4. Obtain the formula $\Phi_{t}$ from $\Phi_{t-1}$ by substituting the value $\sigma\left(x_{t}\right)$ for $x_{t}$ and simplifying (i.e., delete all clauses that got satisfied by assigning $x_{t}$, and omit $x_{t}$ from all other clauses).

5. Return the assignment $\sigma$.

Figure 1: The decimation process.

The analysis performed in [8] is based on an intricate method for directly tracking the execution of BP decimation. Unfortunately this argument does little to illuminate the conceptual reasons for the algorithms' demise. In particular, [8] does not provide a link to the statistical mechanics ideas that inspired the algorithm. The present paper aims to remedy these defects. Here we study the decimation process, an idealized thought experiment that the BP decimation algorithm aims to implement. We show that this experiment undergoes a variety of phase transitions that explain the failure of BP decimation for densities $r>\rho_{0} \cdot 2^{k} / k$. Our results identify phase transitions jointly in terms of the clause/variable density $r$ and with respect to the time parameter of the decimation process. The latter dimension was ignored in the original statistical mechanics work on BP [6, 16] but turns out to have a crucial impact on the performance of the algorithm. On a non-rigorous basis, this has been pointed out recently by Ricci-Tersenghi and Semerjian [19], and our results can be viewed as providing a rigorous version of (substantial parts of) their main results. The results of this paper can also be seen as a generalization of the ones obtained in [1] for random $k$-SAT, and indeed our proofs build upon the techniques developed in that paper.

\section{Results}

BP decimation is a polynomial-time algorithm that aims to (heuristically) implement the 'thought experiment' shown in Fig. 10 [18, 19], which we call the decimation process 1] A moment's reflection reveals that, given a satisfiable input formula $\Phi$, the decimation process outputs a uniform sample from the set of all satisfying assignments of $\Phi$. The obvious obstacle to actually implementing this experiment is the computation of the marginal probability $M_{x_{t}}\left(\Phi_{t-1}\right)$ that $x_{t}$ takes the value 'true' in a random satisfying assignment of $\Phi_{t-1}$, a \#Phard problem in the worst case. Yet the key hypothesis underlying BP decimation is that these marginals can be computed efficiently on random formulas by means of a message passing algorithm. We will return to the discussion of BP decimation and its connection to Experiment 2.1 below.

We are going to study the decimation process when applied to a random formula $\Phi$ for densities $r<2^{k} \ln 2-k$, i.e., in the regime where $\boldsymbol{\Phi}$ is satisfiable w.h.p. More precisely, conditioning on $\boldsymbol{\Phi}$ being satisfiable, we let $\boldsymbol{\Phi}_{t}$ be the (random) formula obtained after running the first $t$ iterations of Experiment 2.1 The variable set of this formula is $V_{t}=\left\{x_{t+1}, \ldots, x_{n}\right\}$, and each clause of $\boldsymbol{\Phi}_{t}$ consists of at most $k$ literals. Let $\mathcal{S}\left(\boldsymbol{\Phi}_{t}\right) \subset\{0,1\}^{V_{t}}$ be the set of all satisfying assignments of $\boldsymbol{\Phi}_{t}$. We say that almost all $\sigma \in \mathcal{S}\left(\boldsymbol{\Phi}_{t}\right)$ have a certain property $\mathcal{A}$ if $\left|\mathcal{A} \cap \mathcal{S}\left(\boldsymbol{\Phi}_{t}\right)\right|=(1-o(1))\left|\mathcal{S}\left(\mathbf{\Phi}_{t}\right)\right|$.

We will identify various phase transition that the formulas $\boldsymbol{\Phi}_{t}$ undergo as $t$ grows from 1 to $n$. As it turns out, these can be characterized via two simple parameters. The first one is the clauses density $r \sim m / n$. Actually, it will be most convenient to work in terms of

$$
\rho=k r / 2^{k},
$$

so that $m / n \sim \rho \cdot 2^{k} / k$. We will be interested in the regime $\rho_{0} \leq \rho \leq k \ln 2$, where $\rho_{0}$ is a constant (independent of $k$ ). The upper bound $k \ln 2$ marks the point where satisfying assignments cease to exist [4]. The second parameter

\footnotetext{
${ }^{1}$ Several different versions of BP decimation have been suggested. In this paper we refer to the simplest but arguably most natural one, also considered in [8, 18, 19]. Other versions decimate the variables in a different order, allowing for slightly better experimental results [6]. 13$]$.
} 
is the fraction

$$
\theta=1-t / n
$$

of 'free' variables (i.e., variables not yet assigned by time $t$ ).

The symmetric phase. Let $\Phi$ be a $k$-CNF on $V$, let $1 \leq t<n$, let $\Phi_{t}$ be the formula obtained after $t$ steps of the decimation process, and suppose that $\sigma \in \mathcal{S}\left(\Phi_{t}\right)$. A variable $x \in V_{t}$ is loose if there is $\tau \in \mathcal{S}\left(\Phi_{t}\right)$ such that $\sigma(x) \neq \tau(x)$ and $d(\sigma, \tau) \leq \ln n$, where $d(\cdot, \cdot)$ denotes the Hamming distance. For any $x \in V_{t}$ we let

$$
M_{x}\left(\Phi_{t}\right)=\frac{\left|\left\{\sigma \in \mathcal{S}\left(\Phi_{t}\right): \sigma(x)=1\right\}\right|}{\left|\mathcal{S}\left(\Phi_{t}\right)\right|}
$$

be the marginal probability that $x$ takes the value 'true' in a random satisfying assignment of $\Phi_{t}$.

Theorem 2.2 There are constants $k_{0}, \rho_{0}>0$ such that for $k \geq k_{0}, \rho_{0} \leq \rho \leq k \ln 2-2 \ln k$, and

$$
k \cdot \theta>\exp \left[\rho\left(1+\frac{\ln \ln \rho}{\rho}+\frac{10}{\rho}\right)\right]
$$

the random formula $\boldsymbol{\Phi}_{t}$ has the following properties w.h.p.

1. In almost all satisfying assignments $\sigma \in \mathcal{S}\left(\boldsymbol{\Phi}_{t}\right)$ at least $0.99 \theta n$ variables are loose.

2. At least $\theta n / 3$ variables $x \in V_{t}$ satisfy $M_{x}\left(\boldsymbol{\Phi}_{t}\right) \in[0.01,0.99]$.

3. The average distance of two random satisfying assignments satisfies

$$
\sum_{\sigma, \tau \in \mathcal{S}\left(\boldsymbol{\Phi}_{t}\right)} d(\sigma, \tau) /\left|\mathcal{S}\left(\boldsymbol{\Phi}_{t}\right)\right|^{2} \geq 0.49 \theta n .
$$

Intuitively, Theorem 2.2 can be summarized as follows. In the early stages of the decimation process (while $\theta$ is 'big'), most variables in a typical $\sigma \in \mathcal{S}\left(\boldsymbol{\Phi}_{t}\right)$ are loose. Hence, the correlations amongst the variables are mostly local: if we 'flip' one variable in $\sigma$, then we can 'repair' the unsatisfied clauses that this may cause by simply flipping another $\ln n$ variables. Furthermore, for at least a good fraction of the variables, the marginals $M_{x}\left(\boldsymbol{\Phi}_{t}\right)$ are bounded away from $0 / 1$. Finally, as the average distance between satisfying assignments is large on average, the set $\mathcal{S}\left(\boldsymbol{\Phi}_{t}\right)$ is 'well spread' over the Hamming cube $\{0,1\}^{V_{t}}$.

Shattering and rigidity. Let $\Phi$ be a $k$-CNF and let $\sigma \in \mathcal{S}\left(\Phi_{t}\right)$. For an integer $\omega \geq 1$ we call a variable $x \in V_{t}$ $\omega$-rigid if any $\tau \in \mathcal{S}\left(\Phi_{t}\right)$ with $\sigma(x) \neq \tau(x)$ satisfies $d(\sigma, \tau) \geq \omega$.

Furthermore, we say that a set $S \subset\{0,1\}^{V_{t}}$ is $(\alpha, \beta)$-shattered if it admits a decomposition $S=\bigcup_{i=1}^{N} R_{i}$ into pairwise disjoint subsets such that the following two conditions are satisfied.

SH1. We have $\left|R_{i}\right| \leq \exp (-\alpha \theta n)|S|$ for all $1 \leq i \leq N$.

SH2. If $1 \leq i<j \leq N$ and $\sigma \in R_{i}, \tau \in R_{j}$, then $\operatorname{dist}(\sigma, \tau) \geq \beta \theta n$.

Theorem 2.3 There are constants $k_{0}, \rho_{0}>0$ such that for $k \geq k_{0}, \rho_{0} \leq \rho \leq k \ln 2-2 \ln k$, and

$$
\frac{\rho}{\ln 2}\left(1+2 \rho^{-2}\right) \leq k \theta \leq \exp \left[\rho\left(1-\frac{\ln \rho}{\rho}-\frac{2}{\rho}\right)\right]
$$

the random formula $\boldsymbol{\Phi}_{t}$ has the following properties w.h.p.

1. In almost all $\sigma \in \mathcal{S}\left(\boldsymbol{\Phi}_{t}\right)$ at least $0.99 \theta n$ variables are $\Omega(n)$-rigid.

2. There exist $\alpha=\alpha(k, \rho)>0, \beta=\beta(k, \rho)>0$ such that $\mathcal{S}\left(\mathbf{\Phi}_{t}\right)$ is $(\alpha, \beta)$-shattered.

3. At least $\theta n / 3$ variables $x \in V_{t}$ satisfy $M_{x}\left(\boldsymbol{\Phi}_{t}\right) \in[0.01,0.99]$.

4. The average distance of two random satisfying assignments is at least $0.49 \theta n$. 
Thus, if the fraction $\theta$ of free variables lies in the regime (1), then in most satisfying $\sigma \in \mathcal{S}\left(\boldsymbol{\Phi}_{t}\right)$ the values assigned to $99 \%$ of the variables are linked via long-range correlations: to 'repair' the damage done by flipping a single rigid variable it is inevitable to reassign a constant fraction of all variables. This is mirrored in the geometry of the set $\mathcal{S}\left(\boldsymbol{\Phi}_{t}\right)$ : it decomposes into exponentially many exponentially tiny subsets, which are mutually separated by a linear Hamming distance $\Omega(n)$. Yet as in the symmetric phase, the marginals of a good fraction of the free variables remain bounded away from $0 / 1$, and the set $\mathcal{S}\left(\boldsymbol{\Phi}_{t}\right)$ remains 'well spread' over the Hamming cube $\{0,1\}^{V_{t}}$.

The condensation phase. Let $\alpha>0$. We say that a set $S \subset\{0,1\}^{\theta n}$ is $\alpha$-condensed if for any $\sigma, \tau \in S$ we have $\operatorname{dist}(\sigma, \tau) \leq \alpha n$.

Theorem 2.4 There are constants $k_{0}, \rho_{0}>0$ such that for $k \geq k_{0}, \rho_{0} \leq \rho \leq k \ln 2-2 \ln k$, and

$$
\ln \rho<k \cdot \theta<\left(1-\rho^{-2}\right) \cdot \rho /(\ln 2)
$$

the random formula $\boldsymbol{\Phi}_{t}$ has the following properties w.h.p.

1. In almost all $\sigma \in \mathcal{S}\left(\boldsymbol{\Phi}_{t}\right)$ at least $0.99 \theta n$ variables are $\Omega(n)$-rigid.

2. The set $\mathcal{S}\left(\boldsymbol{\Phi}_{t}\right)$ is $\exp (2-\rho) / k$-condensed.

3. At least $0.99 \theta n$ variables $x \in V_{t}$ satisfy $M_{x}\left(\boldsymbol{\Phi}_{t}\right) \in\left[0,2^{-k / 2}\right] \cup\left[1-2^{-k / 2}, 1\right]$.

4. There is a set $R \subset V_{t}$ of size $|R| \geq 0.99 \theta n$ such that for any $\sigma, \tau \in \mathcal{S}\left(\boldsymbol{\Phi}_{t}\right)$ we have

$$
|\{x \in R: \sigma(x) \neq \tau(x)\}| \leq k 2^{-k} n \text {. }
$$

In other words, as the decimation process progresses to a point that the fraction $\theta$ of free variables satisfies (2), the set of satisfying assignments shrinks into a condensed subset of $\{0,1\}^{V_{t}}$ of tiny diameter, in contrast to a well-spread shattered set as in Theorem 2.3. Furthermore, most marginals $M_{x}\left(\boldsymbol{\Phi}_{t}\right)$ are either extremely close to 0 or extremely close to 1 . In fact, there is a large set $R$ of variables on which all satisfying assignments virtually agree (more precisely: any two can't disagree on more than $k 2^{-k} n$ variables in $R$ ).

The forced phase. We call a variable $x$ forced in the formula $\Phi_{t}$ if $\Phi_{t}$ has a clause that only contains the variable $x$ (a 'unit clause'). Clearly, in any satisfying assignment $x$ must be assigned so as to satisfy this clause.

Theorem 2.5 There are constants $k_{0}, \rho_{0}>0$ such that for $k \geq k_{0}, \rho_{0} \leq \rho \leq k \ln 2-2 \ln k$, and

$$
1 / n \ll k \cdot \theta<\ln (\rho)(1-10 / \ln \rho)
$$

the random formula $\boldsymbol{\Phi}_{t}$ has the following properties w.h.p.

1. At least $0.99 \theta n$ variables are forced.

2. The set $\mathcal{S}\left(\boldsymbol{\Phi}_{t}\right)$ is $\exp (2-\rho) / k$-condensed.

Belief Propagation. As mentioned earlier, the BP decimation algorithm is an attempt at implementing the decimation process by means of an efficient algorithm. The key issue with this is the computation of the marginals $M_{x_{t}}\left(\Phi_{t-1}\right)$ in step 2 of the decimation process. Indeed, the problem of computing these marginals is \#Phard in the worst case. Thus, instead of working with the 'true' marginals, BP decimation uses certain numbers $\mu_{x_{t}}\left(\Phi_{t-1}, \omega\right)$ that can be computed efficiently, where $\omega \geq 1$ is an integer parameter. The precise definition of the $\mu_{x_{t}}\left(\Phi_{t-1}, \omega\right)$ can be found in Appendix $\mathrm{A}$ (or [6]). Basically, they are the result of a 'local' dynamic programming algorithm ('Belief Propagation') that depends upon the assumption of a certain correlation decay property. For given $k, \rho$, the key hypothesis underpinning the BP decimation algorithm is

Hypothesis 2.6 For any $\varepsilon>0$ there is $\omega=\omega(\varepsilon, k, \rho, n) \geq 1$ such that w.h.p. for all $1 \leq t \leq n$ we have $\left|\mu_{x_{t}}\left(\boldsymbol{\Phi}_{t-1}, \omega\right)-M_{x_{t}}\left(\boldsymbol{\Phi}_{t-1}\right)\right|<\varepsilon$.

In other words, Hypothesis 2.6 states that throughout the decimation process, the 'BP marginals' $\mu_{x_{t}}\left(\Phi_{t-1}, \omega\right)$ are a good approximation to the true marginals $M_{x_{t}}\left(\Phi_{t-1}\right)$. 
Theorem 2.7 There exist constants $c_{0}, k_{0}, \rho_{0}>0$ such that for all $k \geq k_{0}$, and $\rho_{0} \leq \rho \leq k \ln 2-2 \ln k$ the following is true for any integer $\omega=\omega(k, \rho, n) \geq 1$. Suppose that

$$
c_{0} \ln (\rho)<k \cdot \theta<\rho / \ln 2 .
$$

Then for at least $0.99 \theta n$ variables $x \in V_{t}$ we have $\mu_{x}\left(\mathbf{\Phi}_{t}, \omega\right) \in[0.49,0.51]$.

The proof is based on the techniques developed in [8]; the details are omitted from this extended abstract 2 Comparing Theorem 2.4 with Theorem 2.7, we see that w.h.p. for $\theta$ satisfying (4) most of the 'true' marginals $M_{x}\left(\Phi_{t}\right)$ are very close to either 0 or 1 , whereas the 'BP marginals' lie in $[0.49,0.51]$. Thus, in the regime described by (4) the BP marginals do not provide a good approximation to the actual marginals.

Corollary 2.8 There exist constants $c_{0}, k_{0}, \rho_{0}>0$ such that for all $k \geq k_{0}, \rho_{0} \leq \rho \leq k \ln 2-3 \ln k$ Hypothesis 2.6 is untrue.

Summary and discussion. Fix $k \geq k_{0}$ and $\rho \geq \rho_{0}$. Theorems 2.2 2.5 show how the space of satisfying assignments of $\boldsymbol{\Phi}_{t}$ evolves as the decimation process progresses. In the symmetric phase $k \theta \geq \exp \left(\left(1+o_{\rho}(1)\right) \rho\right)$ where there still is a large number of free variables, the correlations amongst the free variables are purely local ('loose variables'). As the number of free variables enters the regime $\left(1+o_{\rho}(1)\right) \rho / \ln 2 \leq k \theta \leq \exp \left(\left(1-o_{\rho}(1)\right) \rho\right)$, the set $\mathcal{S}\left(\boldsymbol{\Phi}_{t}\right)$ of satisfying assignments shatters into exponentially many tiny 'clusters', each of which comprises only an exponentially small fraction of all satisfying assignments. Most satisfying assignments exhibit long-range correlations amongst the possible values that can be assigned to the individual variables ('rigid variables'). This phenomenon goes by the name of dynamic replica symmetry breaking in statistical mechanics [14].

While in the previous phases the set of satisfying assignments is scattered all over the Hamming cube (as witnessed by the average Hamming distance of two satisfying assignments), in the condensation phase (1 $\left.o_{\rho}(1)\right) \ln \rho \leq k \theta \leq\left(1-o_{\rho}(1)\right) \rho / \ln 2$ the set of satisfying assignments has a tiny diameter. This is mirrored by the fact that the marginals of most variables are extremely close to either 0 or 1 . Furthermore, in (most of) this phase the estimates of the marginals resulting from Belief Propagation are off (Theorem 2.7). As part 4 of Theorem 2.4 shows, the mistaken estimates of the Belief Propagation computation would make it impossible for BP decimation to penetrate the condensation phase. More precisely, even if BP decimation would emulate the decimation process perfectly up until the condensation phase commences, with probability $1-\exp (-\Omega(n))$ BP decimation would then assign at least $k 2^{-k} n$ variables in the set $R$ from part 4 of Theorem 2.4 'wrongly' (i.e., differently than they are assigned in any satisfying assignment). In effect, BP decimation would fail to find a satisfying assignment, regardless of its subsequent decisions. Finally, in the forced phase $k \theta \leq\left(1-o_{\rho}(1)\right) \ln \rho$ there is an abundance of unit clauses that make it easy to read off the values of most variables. However, getting stuck in the condensation phase, BP decimation won't reach this regime.

These results suggest that the reason for the failure of BP decimation is the existence of the condensation phase. Intuitively, in the condensation phase the marginals are governed by genuinely global phenomena (essentially expansion properties) that elude the inherently local BP computation. By contrast, it is conceivable that BP does indeed yield the correct marginals in the previous phases. Verifying or falsifying this remains an important open problem.

\section{Related work}

The statistical mechanics perspective. BP/SP decimation are inspired by a generic but highly non-rigorous analysis technique from statistical mechanics called the cavity method [6]. This technique is primarily destined for the analysis of phase transitions. It is based on the (unproven) replica symmetry breaking hypothesis, which aims to characterize the possible types of correlations amongst the variables [14].

In [6, 14] the cavity method was used to study the structure of the set $\mathcal{S}(\Phi)$ of satisfying assignments (or, more accurately, properties of the Gibbs measure) of the undecimated random formula $\boldsymbol{\Phi}$. Thus, the results obtained in that (non-rigorous) work identify phase transitions solely in terms of the formula density $\rho$. On the basis of these results, it was hypothesized that (certain versions of) BP decimation should find satisfying assignments up to $\rho \sim \ln k$ or even up to $\rho \sim k \ln 2$ [14]. The argument given for the latter scenario in [14] is that the key obstacle for BP to approximate the true marginals is condensation. In terms of the parameter $\rho$, the condensation threshold was (non-rigorously) estimated to occur at $\rho=k \ln 2-3 k 2^{-k-1} \ln 2$. However, [8] shows that (the basic version of) BP decimation fails to find satisfying assignments already for $\rho \geq \rho_{0}$, with $\rho_{0}$ a constant independent of $k$.

The explanation for this discrepancy is that [6, 14] neglect the time parameter $\theta=1-t / n$ of the decimation process. As Theorem 2.4 shows, even for fixed $\rho \geq \rho_{0}$ (independent of $k$ ) condensation occurs as the decimation

\footnotetext{
${ }^{2}$ In the appendix we indicate how Theorem 2.7 follows from the results of [8].
} 
process proceeds to $\theta$ in the regime (2). This means that decimating variables has a similar effect on the geometry of the set of satisfying assignments as increasing the clause/variable density. On a non-rigorous basis an analysis both in terms of the formula density $\rho$ and the time parameter $\theta$ was carried out in [19]. Thus, our results can be viewed as a rigorous version of [19] (with proofs based on completely different techniques). In addition, Theorem 2.7 confirms rigorously that for $\rho, \theta$ in the condensation phase, BP does not yield the correct marginals.

The present results have no immediate bearing on the conceptually more sophisticated SP decimation algorithm. However, we conjecture that SP undergoes a similar sequence of phase transitions and that the algorithm will not find satisfying assignments for densities $\rho \geq \rho_{0}$, with $\rho_{0}$ a certain constant independent of $k$.

Rigorous work. Theorem 2.3 can be viewed as a generalization of the results on random $k$-SAT obtained in [1] (which additionally deals with further problems such as random graph/hypergraph coloring). In [1] we rigorously proved a substantial part of the results hypothesized in [14] on shattering and rigidity in terms of the clause/variable density $\rho$; this improved prior work [2, 5, 9]. The new aspect of the present work is that we identify not only a transition for shattering/rigidity, but also for condensation and forcing in terms of both the density $\rho$ and the time parameter $\theta$ of the decimation process. As explained in the previous paragraph, the time parameter is crucial to link these phase transitions to the performance of algorithms such as BP decimation.

In particular, from Theorem 2.3 we can recover the main result of [1] on random $k$-SAT. Namely, if $\rho \geq$ $\ln k+2 \ln \ln k+2$, then (1) is satisfied even for $\theta=1$, i.e., the undecimated random formula $\Phi$ has the properties 1.-4. stated in Theorem 2.3 Technically, the present paper builds upon the methods developed in [1]. In addition, new arguments are needed to accommodate the time parameter $\theta$, to prove the statements on the marginals of the variables in Theorems 2.2 2.4 and to establish the condensation phenomenon (Theorem 2.4).

The best current rigorous algorithmic results for random $k$-SAT are [7, 10, 11, 12]. For general $k$, the best current algorithm succeeds up to $\rho \sim \ln k[7]$.

\section{Analyzing the decimation process}

In the rest of the paper, we are going to sketch the proofs of the main results 3 In this section we perform some groundwork to facilitate a rigorous analysis of the decimation process. The key problem is to get a handle on the following experiment:

D1. Generate a random formula $\Phi$, conditioned on $\Phi$ being satisfiable.

D2. Run the decimation process for $t$ steps to obtain $\boldsymbol{\Phi}_{t}$.

D3. Choose a satisfying assignment $\boldsymbol{\sigma}_{t} \in \mathcal{S}\left(\boldsymbol{\Phi}_{t}\right)$ uniformly at random.

D4. The result is the pair $\left(\boldsymbol{\Phi}_{t}, \boldsymbol{\sigma}_{t}\right)$.

As throughout the paper we only work with densities $m / n$ where $\boldsymbol{\Phi}$ is satisfiable w.h.p., the conditioning in step D1 is essentially void. Recalling that the outcome of the decimation process is a uniformly random satisfying assignment of $\boldsymbol{\Phi}$, we see that the following experiment is equivalent to D1-D4:

U1. Generate a random formula $\Phi$, conditioned on $\boldsymbol{\Phi}$ being satisfiable.

U2. Choose $\sigma \in \mathcal{S}(\boldsymbol{\Phi})$ uniformly at random.

U3. Substitute $\boldsymbol{\sigma}\left(x_{i}\right)$ for $x_{i}$ for $1 \leq i \leq t$ and simplify to obtain a formula $\boldsymbol{\Phi}_{t}$.

U4. The result is the pair $\left(\boldsymbol{\Phi}_{t}, \boldsymbol{\sigma}_{t}\right)$, where $\boldsymbol{\sigma}_{t}: V_{t} \rightarrow\{0,1\}, x \mapsto \boldsymbol{\sigma}(x)$.

Fact 4.1 The two probability distributions induced on formula/assignment pairs by the two experiments D1-D4 and $\mathbf{U 1 - U 4}$ are identical.

Still, an analysis of $\mathbf{U} \mathbf{1}-\mathbf{U} \mathbf{4}$ seems difficult because of $\mathbf{U} 2$ : it is unclear how to analyze (or implement) this step directly. Following [1], we will surmount this problem by considering yet another experiment.

P1. Choose an assignment $\boldsymbol{\sigma}^{\prime} \in\{0,1\}^{V}$ uniformly at random.

P2. Choose a formula $\boldsymbol{\Phi}^{\prime}$ with $m$ clauses that is satisfied by $\boldsymbol{\sigma}^{\prime}$ uniformly at random.

${ }^{3}$ Full proofs can be found in the appendix. 
P3. Substitute $\sigma^{\prime}\left(x_{i}\right)$ for $x_{i}$ for $1 \leq i \leq t$ and simplify to obtain a formula $\boldsymbol{\Phi}_{t}^{\prime}$.

P4. The result is the pair $\left(\boldsymbol{\Phi}_{t}^{\prime}, \boldsymbol{\sigma}_{t}^{\prime}\right)$, where $\boldsymbol{\sigma}_{t}^{\prime}: V_{t} \rightarrow\{0,1\}, x \mapsto \boldsymbol{\sigma}^{\prime}(x)$.

The experiment P1-P4 is easy to implement and, in effect, also amenable to a rigorous analysis. For given the assignment $\boldsymbol{\sigma}^{\prime}$, there are $\left(2^{k}-1\right)\left(\begin{array}{l}n \\ k\end{array}\right)$ clauses in total that evaluate to 'true' under $\boldsymbol{\sigma}^{\prime}$, and to generate $\boldsymbol{\Phi}^{\prime}$ we merely choose $m$ out of these uniformly and independently. Unfortunately, it is not true that the experiment P1-P4 is equivalent to U1-U4. However, we will employ a result from [1] that establishes a connection between these two experiments that is strong enough to extend many results from P1-P4 to U1-U4.

To state this result, observe that P1-P4 and U1-U4 essentially only differ in their first two steps. Thus, let $\Lambda_{k}(n, m)$ denote the set of all pairs $(\Phi, \sigma)$, where $\Phi$ is a $k$-CNF on $V=\left\{x_{1}, \ldots, x_{n}\right\}$ with $m$ clauses, and $\sigma \in \mathcal{S}(\Phi)$. Let $\mathcal{U}_{k}(n, m)$ denote the probability distribution induced on $\Lambda_{k}(n, m)$ by $\mathbf{U} \mathbf{1}-\mathbf{U} \mathbf{2}$, and let $\mathcal{P}_{k}(n, m)$ signify the distribution induced by P1-P2; this distribution is sometimes called the planted model.

Theorem 4.2 ([1]) Suppose $k \geq 4$ and $0<\rho<k \ln 2-k^{2} / 2^{k}$. Let $\mathcal{E} \subset \Lambda_{k}(n, m)$. If $\mathrm{P}_{\mathcal{P}_{k}(n, m)}[\mathcal{E}] \geq$ $1-\exp \left(-\rho n / 2^{k}\right)$ then $\mathrm{P}_{\mathcal{U}_{k}(n, m)}[\mathcal{E}]=1-o(1)$.

\section{Shattering, pairwise distances, and condensation}

To prove shattering and condensation, we adapt arguments from [1, 2, 9] to the situation where we have the two parameters $\theta, \rho$ (rather than just $\rho$ ). Let $\left(\boldsymbol{\Phi}_{t}, \boldsymbol{\sigma}_{t}\right)$ be the (random) outcome of the experiment U1-U4. For $0 \leq \alpha \leq 1$ let $X_{\alpha}\left(\boldsymbol{\Phi}_{t}, \boldsymbol{\sigma}_{t}\right)$ denote the number of satisfying assignments $\tau \in \mathcal{S}\left(\boldsymbol{\Phi}_{t}\right)$ with Hamming distance $d\left(\boldsymbol{\sigma}_{t}, \tau\right)=\alpha \theta n$. To establish the 'shattering' part of Theorem 2.3, we are going to prove the following

Claim 5.1 Under the assumptions of Theorem 2.3 there exist $a_{1}<a_{2}<0.49, a_{3}>0$ depending only on $k, \rho$ such that w.h.p. we have

$$
\begin{aligned}
X_{\alpha}\left(\boldsymbol{\Phi}_{t}, \boldsymbol{\sigma}_{t}\right) & =0 \text { for all } a_{1}<\alpha<a_{2}, \text { and } \\
\max _{\alpha \leq 0.49} X_{\alpha}\left(\boldsymbol{\Phi}_{t}, \boldsymbol{\sigma}_{t}\right) & <\exp \left(-a_{3} n\right) \cdot\left|\mathcal{S}\left(\boldsymbol{\Phi}_{t}\right)\right| .
\end{aligned}
$$

Claim 5.1 implies that for the outcome $\boldsymbol{\Phi}_{t}$ of the first $t$ steps of the decimation process the set $\mathcal{S}\left(\boldsymbol{\Phi}_{t}\right)$ shatters w.h.p. For by Fact 4.1 Claim 5.1 implies that w.h.p. almost all $\sigma_{t} \in \mathcal{S}\left(\boldsymbol{\Phi}_{t}\right)$ are such that (5) and (6) hold. Choose any such $\sigma_{t, 1} \in \mathcal{S}\left(\boldsymbol{\Phi}_{t}\right)$ and let $R_{1}=\left\{\tau \in \mathcal{S}\left(\boldsymbol{\Phi}_{t}\right): d\left(\tau, \sigma_{t, 1}\right) \leq a_{1} n\right\}$. Then, choose $\sigma_{t, 2} \in \mathcal{S}\left(\boldsymbol{\Phi}_{t}\right) \backslash R_{1}$ satisfying (5) and (6), let $R_{2}=\left\{\tau \in \mathcal{S}\left(\boldsymbol{\Phi}_{t}\right) \backslash R_{1}: d\left(\tau, \sigma_{t, 2}\right) \leq a_{1} n\right\}$, and proceed inductively until all remaining satisfying assignments violate either (5) or (6). Let $R_{1}, \ldots, R_{N}$ be the classes constructed in this way and let $R_{0}=\mathcal{S}\left(\boldsymbol{\Phi}_{t}\right) \backslash \bigcup_{i=1}^{N} R_{i}$. An additional (simple) argument is needed to show that $\left|R_{0}\right| \leq \exp (-\Omega(n))\left|\mathcal{S}\left(\boldsymbol{\Phi}_{t}\right)\right|$ w.h.p. The decomposition $R_{0}, \ldots, R_{N}$ witnesses that $\mathcal{S}\left(\boldsymbol{\Phi}_{t}\right)$ shatters.

With respect to pairwise distances of satisfying assignments, (6) implies that w.h.p. only an exponentially small fraction of all satisfying assignments of $\boldsymbol{\Phi}_{t}$ lies within distance $\leq 0.49 \theta n$ of $\boldsymbol{\sigma}_{t}$. It is not difficult to derive the statement made in Theorem 2.3 on the average pairwise distance from this. In addition, the fact that the average pairwise distance of satisfying assignments is $\geq 0.49 \theta n$ w.h.p. implies in combination with a double counting argument the claim about the marginals $M_{x}\left(\boldsymbol{\Phi}_{t}\right)$ in Theorems 2.2 and 2.3

To establish Claim 5.1 we will work with the experiment P1-P4 and use Theorem 4.2 to transfer the result to the experiment $\mathbf{U 1}-\mathbf{U} 4$. Thus, let $\left(\boldsymbol{\Phi}_{t}^{\prime}, \boldsymbol{\sigma}_{t}^{\prime}\right)$ be the (random) outcome of experiment P1-P4, and assume that $k, \rho, \theta$ are as in Theorem 2.3 To prove (5) we need to bound $X_{\alpha}\left(\boldsymbol{\Phi}_{t}^{\prime}, \boldsymbol{\sigma}_{t}^{\prime}\right)$ from above, for which we use the 'first moment method'. Indeed, by standard arguments (similar to those used in [2]) the expectation of $X_{\alpha}\left(\boldsymbol{\Phi}_{t}^{\prime}, \boldsymbol{\sigma}_{t}^{\prime}\right)$ satisfies $\frac{1}{n} \ln \operatorname{E} X_{\alpha}\left(\boldsymbol{\Phi}_{t}^{\prime}, \boldsymbol{\sigma}_{t}^{\prime}\right) \leq \psi(\alpha)$, with

$$
\psi(\alpha)=-\alpha \theta \ln \alpha-(1-\alpha) \theta \ln (1-\alpha)+\frac{2^{k} \rho}{k} \ln \left(1-\frac{1-(1-\alpha \theta)^{k}}{2^{k}-1}\right) .
$$

Thus, in order to prove that $\max _{a_{1}<\alpha<a_{2}} X_{\alpha}\left(\boldsymbol{\Phi}_{t}^{\prime}, \boldsymbol{\sigma}_{t}^{\prime}\right)=0$ w.h.p. we would just have to prove that $\max _{a_{1}<\alpha<a_{2}} \psi(\alpha)<$ 0 (so that Markov's inequality implies that $X_{\alpha}=0$ w.h.p.). But as our goal is to prove a result about the $X_{\alpha}\left(\boldsymbol{\Phi}_{t}, \boldsymbol{\sigma}_{t}\right)$ (i.e., the experiment $\left.\mathbf{U 1}-\mathbf{U} 4\right)$, we need to prove a slightly stronger bound, namely

$$
\max _{a_{1}<\alpha<a_{2}} \psi(\alpha)<-\rho / 2^{k} .
$$


Then Markov's inequality and Theorem 4.2 imply the first part of Claim 5.1. Via elementary calculus, one can show that (7) holds with $a_{1}=\exp (2-\rho)-\varepsilon$ and $a_{2}=\exp (2-\rho)+\varepsilon$ for a sufficiently small $\varepsilon>0$.

To prove (6) we bound $\mathrm{E} X_{\alpha}$ from above by a similar first moment argument. But in addition, we need a lower bound on $\left|\mathcal{S}\left(\boldsymbol{\Phi}_{t}\right)\right|$. To derive this lower bound, we need

Theorem 5.2 ([2]) Assume $k \geq 4$ and $\rho \leq k \ln 2-k^{2} / 2^{k}$. Then w.h.p. $\frac{1}{n} \ln |\mathcal{S}(\boldsymbol{\Phi})| \geq \ln 2+2^{k} \frac{\rho}{k} \ln \left(1-2^{-k}\right)-$ $0.99 \rho / 2^{k}$.

In combination with a double counting argument, Theorem 5.2 implies the following lower bound on $\mathcal{S}\left(\boldsymbol{\Phi}_{t}\right)$, which entails the second part of Claim 5.1

Corollary 5.3 Let $\left(\boldsymbol{\Phi}_{t}, \boldsymbol{\sigma}_{t}\right)$ be the outcome of $\mathbf{U 1 - U 4}$. Let $1 \leq t \leq n$. Then w.h.p. $\frac{1}{n} \ln \left|\mathcal{S}\left(\boldsymbol{\Phi}_{t}\right)\right| \geq \theta \ln 2+$ $2^{k} \frac{\rho}{k} \ln \left(1-2^{-k}\right)-\frac{\rho}{2^{k}}$.

The proof of the 'condensation' part of Theorem 2.4 is based on similar arguments. Basically, to show condensation we need to prove that $\max _{\alpha>a_{4}} X_{\alpha}\left(\boldsymbol{\Phi}_{t}, \boldsymbol{\sigma}_{t}\right)<0$ with probability $1-\exp (-\Omega(n))$, where we let $a_{4}=\exp (2-\rho)$. This is done via the first moment method and boils down to proving that $\psi(\alpha)<-\rho / 2^{k}$ for all $\alpha>a_{4}$.

\section{Rigid variables}

Assume that $k, \rho, \theta$ satisfy the assumptions of Theorem 2.3. Let $\left(\boldsymbol{\Phi}_{t}, \boldsymbol{\sigma}_{t}\right)$ be the (random) outcome of U1-U4. Our goal is to show that w.h.p. most variables $x \in V_{t}$ are rigid.

What is the basic obstacle that makes it difficult to 'flip' the value of $x$ ? Observe that we can simply assign $x$ the opposite value $1-\boldsymbol{\sigma}_{t}(x)$, unless $\boldsymbol{\Phi}_{t}$ has a clause $\mathcal{C}$ in which either $x$ or $\bar{x}$ is the only literal that is true under $\sigma_{t}$. If there is such a clause, we say that $x \operatorname{supports} \mathcal{C}$. But even if $x$ supports a clause $\mathcal{C}$ it might be easy to flip. For instance, if $\mathcal{C}$ features some variable $y \neq x$ that does not support a clause, then we could just flip both $x, y$ simultaneously. Thus, to establish the existence of $\Omega(n)$-rigid variables we need to analyze the distribution of the number of clauses that a variable supports, the probability that these clauses only consists of variables that support further clauses, the probability that the same is true of those clauses, etc.

This analysis can be performed fairly neatly for the outcome $\left(\boldsymbol{\Phi}_{t}^{\prime}, \boldsymbol{\sigma}_{t}^{\prime}\right)$ of the experiment $\mathbf{P 1}-\mathbf{P 4}$. Let us sketch how this works, and why rigidity occurs at $k \theta=\exp ((1+o(1)) \rho)$ (cf. (1)). For a variable $x \in V_{t}$ we let $S_{x}$ be the number of clauses supported by $x$. Given the assignment $\boldsymbol{\sigma}^{\prime}$ chosen in step P1, there are a total of $\left(\begin{array}{c}n-1 \\ k-1\end{array}\right)$ possible clauses that $x$ supports. Since in step $\mathbf{P} 2$ we include $m$ out of the $\left(2^{k}-1\right)\left(\begin{array}{l}n \\ k\end{array}\right)$ possible clauses satisfied under $\boldsymbol{\sigma}^{\prime}$ uniformly and independently, we get

$$
\mathrm{E}\left[S_{x}\right]=m\left(\begin{array}{l}
n-1 \\
k-1
\end{array}\right)\left(\left(2^{k}-1\right)\left(\begin{array}{l}
n \\
k
\end{array}\right)\right)^{-1}=\rho /\left(1-2^{-k}\right) \geq \rho .
$$

In fact, $S_{x}$ is binomially distributed. Hence, $\mathrm{P}\left[S_{x}=0\right] \leq \exp (-\rho)$. Thus, the expected number of variables $x \in V_{t}$ with $S_{x}=0$ is $\leq \theta n \exp (-\rho)$. Furthermore, if we condition on $S_{x}=j \geq 1$, then the actual clauses $\mathcal{C}_{1}, \ldots, \mathcal{C}_{j}$ supported by $x$ are just independently uniformly distributed over the set of all $\left(\begin{array}{l}n-1 \\ k-1\end{array}\right)$ possible clauses that $x$ supports. Therefore, the expected number of variables $y \in V_{t}$ with $S_{y}=0$ occurring in one of these clauses $\mathcal{C}_{i}$ is $(1+o(1))(k-1) \cdot \theta \exp (-\rho) \leq k \theta \exp (-\rho)$. Hence, if $\theta$ is as in (1), then this number is $\leq \exp (-2) / \rho$, i.e., 'small' for $\rho \geq \rho_{0}$ sufficiently big. Thus, we would expect that most clauses supported by $x$ indeed consist exclusively of variables that support other clauses. This heuristic argument shows that for $\theta$ as in (1) we can plausibly expect most variables to be rigid.

Let us now indicate how this argument can be carried out in detail. Analyzing the distribution of the variables $S_{x}$ in the experiment P1-P4 and extending the result to the experiment U1-U4 via Theorem 4.2, and setting $\zeta=\rho^{2} / \exp (\rho)$, we obtain the following.

Proposition 6.1 Suppose that $k, \rho, \theta$ satisfy the assumptions of Theorem 2.3. Then w.h.p. in a random pair $\left(\boldsymbol{\Phi}_{t}, \boldsymbol{\sigma}_{t}\right)$ generated by the experiment $\mathbf{U 1} \mathbf{\mathbf { U } 4}$ no more than $2 \zeta \theta n$ variables in $V_{t}$ support fewer than three clauses,

To establish rigidity, we need to show that most variables support clauses in which only variables occur that support other clauses. To express this, we say that $S \subset V_{t}$ is $t$-self-contained if each $x \in S$ supports at least two clauses of $\boldsymbol{\Phi}_{t}$ that contain variables from $S$ only. From Proposition 6.1 we can derive the following. 
Proposition 6.2 Suppose that $k, \rho, \theta$ satisfy the assumptions of Theorem 2.3 The outcome $\left(\boldsymbol{\Phi}_{t}, \boldsymbol{\sigma}_{t}\right)$ of $\mathbf{U 1}-\mathbf{U} \mathbf{4}$ has a t-self-contained set of size $(1-3 \zeta) \theta n$ w.h.p.

Suppose that $\left(\boldsymbol{\Phi}_{t}, \boldsymbol{\sigma}_{t}\right)$ has a self-contained set $S$ of size $(1-3 \zeta) \theta n$. To flip the value of a variable $x \in S$ we need to also flip one other variable from each of the (at least two) clauses that $x$ supports and that consist of variables from $S$ only. As each of these two variables, in turn, supports at least two clauses comprised of variables from $S$ only, we need to also flip further variables in those. But these variables are again contained in $S$. This suggests that attempting to flip $x$ will entail an avalanche of further flips. Indeed, the expansion properties of the random formula $\boldsymbol{\Phi}_{t}$ imply the following.

Proposition 6.3 Suppose that $k, \rho, \theta$ satisfy the assumptions of Theorem 2.3 There is $\chi=\chi(k, \rho)>0$ such that the outcome $\left(\boldsymbol{\Phi}_{t}, \boldsymbol{\sigma}_{t}\right)$ of $\mathbf{U 1 - U} \mathbf{4}$ has the following property w.h.p.: all variables that are contained in a $t$-self-contained set are $\chi n$-rigid.

Propositions 6.2 and 6.3 directly imply part 1 of Theorem 2.3 Self-contained sets also play a key role in the proof of Theorem 2.4 Propositions 6.2 and 6.3 can be extended to the regime of $\theta$ as in Theorem 2.4 and the set $R$ in part 4 of that theorem is simply a $t$-self-contained set. Expansion properties of the random formula together with the bound on the diameter of the set $\mathcal{S}\left(\boldsymbol{\Phi}_{t}\right)$ of satisfying assignments from part 2 of Theorem 2.4 imply that there are no two satisfying assignments that disagree on more than $k n / 2^{k}$ variables from $R$. In combination with a double-counting argument, this implies the statement on the marginals in part 3 of Theorem 2.4 . Finally, the claim about forced variables in Theorem 2.5 can be proved via a similar (but simpler) argument as sketched in this section.

\section{References}

[1] D. Achlioptas, A. Coja-Oghlan: Algorithmic barriers from phase transitions. Proc. 49th FOCS (2008) 793802.

[2] D. Achlioptas, A. Coja-Oghlan, F. Ricci-Tersenghi: On the solution space geometry of random formulas. Random structures and algorithms, awaiting publication.

[3] D. Achlioptas, C. Moore: Random $k$-SAT: two moments suffice to cross a sharp threshold. SIAM Journal on Computing 36 (2006) 740-762.

[4] D. Achlioptas, Y. Peres: The threshold for random $k$-SAT is $2^{k} \ln 2-O(k)$. Journal of the AMS 17 (2004) 947-973.

[5] D. Achlioptas, F. Ricci-Tersenghi: Random formulas have frozen variables. SIAM J. Comput. 39 (2009) 260-280.

[6] A. Braunstein, M. Mézard, R. Zecchina: Survey propagation: an algorithm for satisfiability. Random Structures and Algorithms 27 (2005) 201-226.

[7] A. Coja-Oghlan: A better algorithm for random k-SAT. SIAM J. Computing 39 (2010) 2823-2864.

[8] A. Coja-Oghlan: On belief propagation guided decimation for random $k$-SAT. Proc. 22nd SODA (2011) 957-966.

[9] H. Daudé, M. Mézard, T. Mora, R. Zecchina: Pairs of SAT-assignments in random Boolean formulae. Theoretical Computer Science 393 (2008) 260-279.

[10] A. Frieze, S. Suen: Analysis of two simple heuristics on a random instance of $k$-SAT. Journal of Algorithms 20 (1996) 312-355.

[11] M. Hajiaghayi, G. Sorkin: The satisfiability threshold of random 3-SAT is at least 3.52. IBM Research Report RC22942 (2003).

[12] A. Kaporis, L. Kirousis, E. Lalas: The probabilistic analysis of a greedy satisfiability algorithm. Random Structures and Algorithms 28 (2006) 444-480. 
[13] L. Kroc, A. Sabharwal, B. Selman: Message-passing and local heuristics as decimation strategies for satisfiability. Proc 24th SAC (2009) 1408-1414.

[14] F. Krzakala, A. Montanari, F. Ricci-Tersenghi, G. Semerjian, L. Zdeborova: Gibbs states and the set of solutions of random constraint satisfaction problems. Proc. National Academy of Sciences 104 (2007) 1031810323.

[15] S. Mertens, M. Mézard, R. Zecchina: Threshold values of random $K$-SAT from the cavity method. Random Struct. Alg. 28 (2006) 340-373.

[16] M. Mézard, G. Parisi, R. Zecchina: Analytic and algorithmic solution of random satisfiability problems. Science 297 (2002) 812-815.

[17] D. Mitchell, B. Selman, H. Levesque: Hard and easy distribution of SAT problems. Proc. 10th AAAI (1992) 459-465.

[18] A. Montanari, F. Ricci-Tersenghi, G. Semerjian: Solving constraint satisfaction problems through Belief Propagation-guided decimation. Proc. 45th Allerton (2007).

[19] F. Ricci-Tersenghi, G. Semerjian: On the cavity method for decimated random constraint satisfaction problems and the analysis of belief propagation guided decimation algorithms. J. Stat. Mech. (2009) P09001. 


\section{Appendix}

Appendix A contains a discussion of Belief Propagation. The remaining appendices contain the full proofs of the results stated in Section 2 Appendix B gives an overview of how the proofs are organized.

\section{A Detailed description of BP decimation}

The BP decimation algorithm can be viewed as an attempt at implementing the decimation process (Experiment 2.1. . As mentioned earlier, the key issue with this is the computation (or approximation) of the the marginals $M_{x}\left(\Phi_{t-1}\right)$. BP decimation basically tries to approximate these marginals by means of a 'local' computation.

For clearly, the marginals $M_{x}\left(\Phi_{t-1}\right)$ are influenced by 'local' effects. For instance, if $x$ occurs in a unit clause $a$ of $\Phi_{t-1}$, i.e., a clause of length one, then $x$ must be assigned so as to satisfy $a$. Hence, if $x$ appears in a positively, then $M_{x}\left(\Phi_{t-1}\right)=1$, and otherwise $M_{x}\left(\Phi_{t-1}\right)=0$. Similarly, if $x$ occurs only positively in $\Phi_{t-1}$, then $M_{x}\left(\Phi_{t-1}\right) \geq 1 / 2$. More intricately, if $x$ occurs in a clause $a$ that contains another variable $y$ that appears is a unit clause $b$, then this will affect the marginal of $x$.

The key hypothesis underlying BPdec is that in random formulas such local effects determine the marginals $M_{x}\left(\Phi_{t-1}\right)$ asymptotically. To define 'local' precisely, we need a metric on the variables/clauses. This metric is the one induced by the factor graph $G=G\left(\Phi_{t-1}\right)$ of $\Phi_{t-1}$, which is a bipartite graph whose vertices are the variables $V_{t-1}=\left\{x_{t}, \ldots, x_{n}\right\}$ and the clauses of $\Phi_{t-1}$. Each clause is adjacent to the variables that occur in it. For an integer $\omega \geq 1$ let $N^{\omega}\left(x_{t}\right)$ signify the set of all vertices of $G$ that have distance at most $2 \omega$ from $x_{t}$. Then the induced subgraph $G\left[N^{\omega}\left(x_{t}\right)\right]$ corresponds to the sub-formula of $\Phi_{t-1}$ obtained by removing all clauses and variables at distance more than $2 \omega$ from $x_{t}$. Note that all vertices at distance precisely $2 \omega$ are variables, so that any satisfying assignment of $\Phi$ induces a satisfying assignment of the sub-formula. Let us denote by $M_{x_{t}}\left(\Phi_{t-1}, \omega\right)$ the marginal probability that $x_{t}$ takes the value 1 in a random satisfying assignment of this sub-formula.

Of course, for a worst-case formula $\Phi$ the 'local' marginals $M_{x_{t}}\left(\Phi_{t-1}, \omega\right)$ may be just as difficult to compute as the overall marginals $M_{x_{t}}\left(\Phi_{t-1}\right)$ themselves. Therefore, BP decimation employs an efficient dynamic programming heuristic called Belief Propagation ('BP'), which yields certain values $\mu_{x_{t}}\left(\Phi_{t-1}, \omega\right) \in[0,1]$; we will state this heuristic below. If the induced subgraph $G\left[N^{\omega}\left(x_{t}\right)\right]$ is a tree, then indeed $\mu_{x_{t}}\left(\Phi_{t-1}, \omega\right)=M_{x_{t}}\left(\Phi_{t-1}, \omega\right)$. Moreover, standard arguments show that in a random formula $\Phi$ actually $G\left[N^{\omega}\left(x_{t}\right)\right]$ is a tree w.h.p. so long as $\omega=o(\ln n)$. Of course, more generally, in order to obtain an efficient algorithm it would be sufficient for the BP outcomes $\mu_{x_{t}}\left(\Phi_{t-1}, \omega\right)$ to approximate the true overall marginals $M_{x_{t}}\left(\Phi_{t-1}\right)$ well for some polynomially computable and polynomially bounded function $\omega=\omega(n) \geq 1$.

To define the numbers $\mu_{x_{t}}\left(\Phi_{t-1}, \omega\right)$ formally, we need to define Belief Propagation for $k$-SAT. To this end, let $N(v)$ denote the neighborhood of a vertex $v$ of the factor graph $G\left(\Phi_{t-1}\right)$. For a variable $x \in V_{t}$ and a clause $a \in N(x)$ we will denote the ordered pair $(x, a)$ by $x \rightarrow a$. Similarly, $a \rightarrow x$ stands for the pair $(a, x)$. Furthermore, we let $\operatorname{sign}(x, a)=1$ if $x$ occurs in $a$ positively, and $\operatorname{sign}(x, a)=-1$ otherwise.

The message space $M\left(\Phi_{t-1}\right)$ is the set of all tuples $\left(\mu_{x \rightarrow a}(\zeta)\right)_{x \in V_{t}, a \in N(x), \zeta \in\{0,1\}}$ such that $\mu_{x \rightarrow a}(\zeta) \in[0,1]$ and $\mu_{x \rightarrow a}(0)+\mu_{x \rightarrow a}(1)=1$ for all $x, a, \zeta$. For $\mu \in M(\Phi)$ we define $\mu_{a \rightarrow x}(\zeta)=1$ if $\zeta=(1+\operatorname{sign}(x, a)) / 2$, and

$$
\mu_{a \rightarrow x}(\zeta)=1-\prod_{y \in N(a) \backslash\{x\}} \mu_{y \rightarrow a}\left(\frac{1-\operatorname{sign}(y, a)}{2}\right)
$$

otherwise. Furthermore, we define the belief propagation operator BP as follows: for any $\mu \in M\left(\Phi_{t-1}\right)$ we define $\operatorname{BP}(\mu) \in M\left(\Phi_{t-1}\right)$ by letting

$$
(\operatorname{BP}(\mu))_{x \rightarrow a}(\zeta)=\frac{\prod_{b \in N(x) \backslash\{a\}} \mu_{b \rightarrow x}(\zeta)}{\prod_{b \in N(x) \backslash\{a\}} \mu_{b \rightarrow x}(0)+\prod_{b \in N(x) \backslash\{a\}} \mu_{b \rightarrow x}(1)}
$$

unless the denominator equals zero, in which case $(\mathrm{BP}(\mu))_{x \rightarrow a}(\zeta)=\frac{1}{2}$.

Finally, the values $\mu_{x}\left(\Phi_{t}, \omega\right)$ are defined as follows. Let $\mu[0]=\frac{1}{2} \cdot \mathbf{1} \in M\left(\Phi_{t-1}\right)$ be the vector with all 


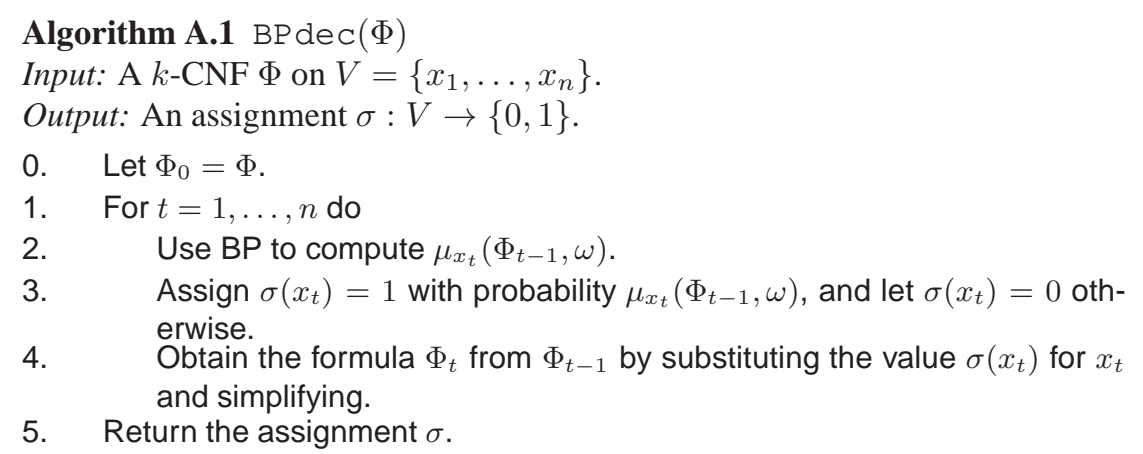

Figure 2: The BP decimation algorithm.

entries equal to $\frac{1}{2}$. Moreover, define inductively $\mu[\ell]=\operatorname{BP}(\mu[\ell-1])$ for $1 \leq \ell \leq \omega$. Then

$$
\mu_{x}\left(\Phi_{t-1}, \omega\right)=\frac{\prod_{b \in N(x)} \mu_{b \rightarrow x}(1)[\omega]}{\prod_{b \in N(x)} \mu_{b \rightarrow x}(0)[\omega]+\prod_{b \in N(x)} \mu_{b \rightarrow x}(1)[\omega]}
$$

for any $x \in V_{t}$, unless the denominator is zero, in which case we set $\mu_{x}\left(\Phi_{t-1}, \omega\right)=\frac{1}{2}$.

The intuition here is that the $\mu_{x \rightarrow a}(\zeta)$ are 'messages' from a variable $x$ to the clauses $a$ in which $x$ occurs, indicating how likely $x$ were to take the value $\zeta$ if clause $a$ were removed from the formula. Based on these, (8) yields messages $\mu_{a \rightarrow x}(\zeta)$ from clauses $a$ to variables $x$, indicating the probability that $a$ is satisfied if $x$ takes the value $\zeta$ and all other variables $y \in N(a) \backslash\{x\}$ are assigned independently to either value $\xi \in\{0,1\}$ with probability $\mu_{y \rightarrow a}(\xi)$. The BP operator (9) then uses these messages $\mu_{a \rightarrow x}$ in order to 'update' the messages from variables to clauses. More precisely, for each $x$ and $a \in N(x)$ the new messages $(\operatorname{BP}(\mu))_{x \rightarrow a}(\zeta)$ are computed under the hypothesis that all other clauses $b \in N(x) \backslash\{a\}$ are satisfied with probabilities $\mu_{b \rightarrow x}(\zeta)$ independently if $x$ takes the value $\zeta$. Finally, the difference between (9) and (10) is that the latter product runs over all clauses $b \in N(x)$. An inductive proof shows that, if for a variable $x$ the subgraph $G\left[N^{\omega}(x)\right]$ of the factor graph is a tree, then in fact $\mu_{x}\left(\Phi_{t}, \omega\right)=M_{x}\left(\Phi_{t}, \omega\right)[\overline{6}$. Figure 2 shows the BP decimation algorithm.

\section{B Overview}

In Section 2 we described the main results of this paper arranged according to the various phases that the decimation process passes through. But to prove these results, it is necessary to proceed in a different order. To facilitate this, we will state the main results in the order in which the proofs proceed. We begin with the statements on the loose/rigid/forced variables.

Theorem B.1 There exist constants $k_{0}, \rho_{0}>0$ such that for all $k \geq k_{0}$ and $\rho_{0} \leq \rho \leq k \ln 2-2 \ln k$ the following three statements hold for a random pair $\left(\boldsymbol{\Phi}_{t}, \sigma_{t}\right)$ chosen from the experiment $\mathbf{U 1}-\mathbf{U} \mathbf{4}$ w.h.p.

1. If $k \theta>\exp \left[\rho\left(1+\frac{\ln \ln \rho}{\rho}+\frac{10}{\rho}\right)\right]$, then at least $0.99 \theta n$ variables $x \in V_{t}$ are loose w.h.p.

2. If $1<k \theta<\exp \left[\rho\left(1-\frac{3 \ln \rho}{\rho}\right)\right]$, then at least $\rho^{3} \exp (-\rho) \theta n$ variables $x \in V_{t}$ are $\Omega(n)$-rigid w.h.p.

3. If $\ln (n) / n<\theta<(\ln (\rho)-10) / k$, then at least $0.99 \theta n$ variables are forced w.h.p.

The second type of statement concerns the global structure of the set of satisfying assignments, summarized in the following theorem.

Theorem B.2 There exist constants $k_{0}, \rho_{0}>0$ such that for all $k \geq k_{0}$, and $\rho_{0} \leq \rho \leq k \ln 2-2 \ln k$ the following three statements hold. 
1. If

$$
\frac{\rho}{\ln 2}\left(1+\rho^{-2}+2^{2-k}\right) \leq k \theta \leq \exp \left[\rho\left(1-\frac{\ln \rho}{\rho}-\frac{2}{\rho}\right)\right]
$$

then $\mathcal{S}\left(\boldsymbol{\Phi}_{t}\right)$ is $(\exp (2-\rho)-\varepsilon, \exp (2-\rho)+\varepsilon)$-shattered w.h.p. for some $\varepsilon=\varepsilon(k, \rho)>0$.

2. If $\theta<(\rho-1 / \rho) /(k \ln 2)$, then $\mathcal{S}\left(\boldsymbol{\Phi}_{t}\right)$ is $\exp (2-\rho)$-condensed w.h.p.

3. If $\theta>\rho\left(1+2 / \rho^{2}\right) /(k \ln 2)$, then the average distance between two random elements of $\mathcal{S}\left(\boldsymbol{\Phi}_{t}\right)$ is at least $0.49 \theta n$ w.h.p.

The next theorem contains the statements about the marginals of the truth values of individual variables.

Theorem B.3 There exist constants $k_{0}, \rho_{0}>0$ such that for all $k \geq k_{0}$, and $\rho_{0} \leq \rho \leq k \ln 2-2 \ln k$ the following two statements hold.

1. If $\theta \geq \frac{\rho}{k \ln 2}\left(1+1 / \rho^{2}+k / 2^{k-2}\right)$, then w.h.p. for at least $\theta n / 3$ variables $x \in V_{t}$ we have

$$
M_{x}\left(\boldsymbol{\Phi}_{t}\right) \in[0.01,0.99] .
$$

2. If $\ln (n) / n<\theta<\rho\left(1-1 / \rho^{2}\right) /(k \ln 2)$, then w.h.p. for all but $\exp (-\rho) \theta n$ variables $x \in V_{t}$ we have

$$
M_{x}\left(\boldsymbol{\Phi}_{t}\right) \in\left[0,2^{-k / 2}\right] \cup\left[1-2^{-k / 2}, 1\right] .
$$

Theorems 2.2 2.5 follow directly from Theorems B.1 B.3 by reordering the individual statements according to the phases they appear in, apart from part 4 of Theorem 2.4 whose proof is given in Appendix F.2. After stating some preliminaries in Appendix C, we will prove Theorem B.1 in Appendix D Then, in Appendix E we will prove Theorem B.2. Further, Appendix $\mathbf{E}$ contains the proof of Theorem B.3 Finally, in Appendix G we prove Theorem 2.7

\section{Preliminaries}

Recall that $V_{t}=\left\{x_{t+1}, \ldots, x_{n}\right\}$. In addition, we let $L_{t}=\left\{x_{t+1}, \bar{x}_{t+1}, \ldots, x_{n}, \bar{x}_{n}\right\}$. For a literal $l$ let $|l|$ be the underlying variable. For a formula $\Phi$ on $V=\left\{x_{1}, \ldots, x_{n}\right\}$, an assignment $\sigma \in\{0,1\}^{V}$, and $1 \leq t \leq n$ we let $\Phi_{t, \sigma}$ denote the formula obtained by substituting $\sigma\left(x_{s}\right)$ for $x_{s}$ for all $1 \leq s \leq t$ and simplifying.

We need the following Chernoff bound on the tails of a binomially distributed random variable $X$ with mean $\lambda$ : for any $t>0$

$$
\mathrm{P}(X \geq \lambda+t) \leq \exp (-t \cdot \varphi(t / \lambda)) \quad \text { and } \quad \mathrm{P}(X \leq \lambda-t) \leq \exp (-t \cdot \varphi(-t / \lambda)) .
$$

where

$$
\varphi(x)=(1+x) \ln (1+x)-x .
$$

We will need the following consequence of Theorem 5.2 ( $(\mathrm{cf}$. Corollary 5.3 in the main part of the paper).

Corollary C.1 Let $1 \leq t \leq n$. Let $\left(\Phi_{t}, \sigma_{t}\right)$ be a pair chosen from the experiment $\mathbf{U} 1-\mathbf{U} 4$. Then w.h.p.

$$
\frac{1}{n} \ln \left|\mathcal{S}\left(\Phi_{t}\right)\right| \geq(1-t / n) \ln 2+r \ln \left(1-2^{-k}\right)-\frac{k r}{4^{k}} .
$$

Proof. Let $\Phi$ be a formula such that $\frac{1}{n} \ln |\mathcal{S}(\Phi)| \geq \ln 2+r \ln \left(1-2^{-k}\right)-k r / 4^{k}$. By Theorem 5.2 the random formula $\Phi$ has this property w.h.p. Thus, it suffices to show that for a random $\sigma \in \mathcal{S}(\Phi)$ the bound (13) holds w.h.p. To this end, let $\mathcal{I}=\{0,1\}^{t}$. Moreover, for each $\sigma \in\{0,1\}^{n}$ let $\left.\sigma\right|_{t}$ be the vector $\left(\sigma\left(x_{1}\right), \ldots, \sigma\left(x_{t}\right)\right) \in \mathcal{I}$. For each $\sigma_{*} \in \mathcal{I}$ let $Z\left(\sigma_{*}\right)$ be the number of assignments $\sigma \in \mathcal{S}(\Phi)$ such that $\left.\sigma\right|_{t}=\sigma_{*}$. If $\sigma \in \mathcal{S}(\Phi)$ is chosen uniformly at random, then for any $\sigma_{*} \in \mathcal{I}$ we have

$$
\mathrm{P}\left[\left.\sigma\right|_{t}=\sigma_{*}\right]=Z\left(\sigma_{*}\right) / Z, \text { where } Z=\sum_{\tau \in \mathcal{I}} Z(\tau)=|\mathcal{S}(\Phi)| .
$$


Let $\xi>0$ be a sufficiently small number and let

$$
q=\mathrm{P}\left[Z\left(\left.\sigma\right|_{t}\right)<\exp (-t \ln 2-\xi n) \cdot Z\right],
$$

where $\sigma \in \mathcal{S}(\Phi)$ is chosen uniformly at random. Then

$$
q=\sum_{\sigma_{*} \in \mathcal{I}: Z\left(\sigma_{*}\right) \leq \frac{Z}{\exp (\xi n+t \ln 2)}} Z\left(\sigma_{*}\right) / Z \leq \frac{2^{t}}{Z} \cdot \frac{Z}{\exp (\xi n+t \ln 2)} \leq \exp (-\xi n),
$$

whence the assertion follows.

In Section 4 we introduced the experiment P1-P4, which led to the planed model $\mathcal{P}_{k}(n, m)$. In addition, we need the following variant of the planted model.

P1'. Choose an assignment $\boldsymbol{\sigma}^{\prime} \in\{0,1\}^{V}$ uniformly at random.

P2'. Choose a formula $\boldsymbol{\Phi}^{\prime}$ by including each of the $\left(2^{k}-1\right)\left(\begin{array}{l}n \\ k\end{array}\right)$ possible clauses that are satisfied under $\boldsymbol{\sigma}^{\prime}$ with probability $p=m /\left(\left(2^{k}-1\right)\left(\begin{array}{l}n \\ k\end{array}\right)\right)$ independently.

P3'. Substitute $\boldsymbol{\sigma}^{\prime}\left(x_{i}\right)$ for $x_{i}$ for $1 \leq i \leq t$ and simplify to obtain a formula $\boldsymbol{\Phi}_{t}^{\prime}$.

P4'. The result is the pair $\left(\boldsymbol{\Phi}_{t}^{\prime}, \boldsymbol{\sigma}_{t}^{\prime}\right)$, where $\boldsymbol{\sigma}_{t}^{\prime}: V_{t} \rightarrow\{0,1\}, x \mapsto \boldsymbol{\sigma}^{\prime}(x)$.

Steps P1'-P2' of this experiment induce a probability distribution $\mathcal{P}_{k}^{\prime}(n, m)$ on formula/assignment pairs. The following corollary establishes a connection between this distribution and the distribution $\mathcal{U}_{k}(n, m)$.

Corollary C.2 ([1]) Suppose that $k \geq 4$ and $0<r<2^{k} \ln 2-k$. Let $\mathcal{E}$ be any property of formula/assignment pairs. If $\mathrm{P}_{\mathcal{P}_{k}^{\prime}(n, m)}[\mathcal{E}] \geq 1-\exp \left(-k r n / 4^{k}\right)$ then $\mathrm{P}_{\mathcal{U}_{k}(n, m)}[\mathcal{E}]=1-o(1)$.

We will need the following elementary observation about the distribution $\mathcal{P}_{k}^{\prime}(n, m)$.

Lemma C.3 Let $(\Phi, \sigma)$ be a pair chosen from the distribution $\mathcal{P}_{k}^{\prime}(n, m)$.

1. For each literal $l$ that is true under $\sigma$ the number of clauses supported by $l$ is binomially distributed $\operatorname{Bin}\left(\frac{k}{n}\right.$. $\left.\left(\begin{array}{l}n \\ k\end{array}\right), m /\left(\left(2^{k}-1\right)\left(\begin{array}{l}n \\ k\end{array}\right)\right)\right)$.

2. For any integer $D$ the number of literals $l$ that support fewer than $D$ clauses is binomially distributed with mean

$$
n \cdot \mathrm{P}\left[\operatorname{Bin}\left(\frac{k}{n} \cdot\left(\begin{array}{l}
n \\
k
\end{array}\right), \frac{m}{\left(2^{k}-1\right)\left(\begin{array}{l}
n \\
k
\end{array}\right)}\right)<D\right] .
$$

Proof. Without loss of generality we may condition on $\sigma$ assigning the value true to all variables. For any variable $x$ let $\mathcal{S}_{x}$ be the set of all possible clauses in which $x$ is the only positive literal. Then $\left|\mathcal{S}_{x}\right|=k\left(\begin{array}{l}n-1 \\ k-1\end{array}\right)=\frac{k}{n} \cdot\left(\begin{array}{l}n \\ k\end{array}\right)$. (First choose one of the $k$ slots where to place $x$, then choose the $k-1$ other variables occurring in the clause; the signs are prescribed by $x$ being the unique positive literal.) Moreover, let $S_{x}$ be the number of clauses from $\mathcal{S}_{x}$ that actually appear in the random formula $F$. As each of the clauses in $\mathcal{S}_{x}$ is included in $F$ with probability $p=m /\left(\left(2^{k}-1\right)\left(\begin{array}{l}n \\ k\end{array}\right)\right)$ independently, $S_{x}$ has a binomial distribution $\operatorname{Bin}\left(\frac{k}{n} \cdot\left(\begin{array}{l}n \\ k\end{array}\right), p\right)$. This establishes 1 .

Since for any two variables $x, y$ we have $\mathcal{S}_{x} \cap \mathcal{S}_{y}=\emptyset$, the random variables $S_{x}$ are mutually independent for all variables $x$. Therefore, the number $S=\sum_{x} 1_{\left\{S_{x}<D\right\}}$ of variables supporting fewer than $D$ clauses in $F$ is binomially distributed as well.

There is a natural way to associate a bipartite graph with a $k$-CNF $\Phi$, known as the factor graph. Its vertices are the variables and the clauses of $\Phi$, and each clause is adjacent to all the variables it contains. For a variable $x$ we let $N_{3}(x)$ be the subgraph of that is spanned by all vertices at distance at most 3 from $x$. A variable $x$ is tame if $N_{3}(x)$ is acyclic and contains no more than $\ln (n)$ variables. The following is a well-known fact about random $k$-CNFs.

Proposition C.4 Suppose that $k \geq 3$ and $0<r \leq 2^{k} \ln 2$. W.h.p. all but o(n) variables are tame in $\boldsymbol{\Phi}$.

Finally, the following lemma expresses an elementary 'expansion property' of the random formula $\boldsymbol{\Phi}$. 
Lemma C.5 There is a number $\chi=\chi(k)>0$ such that for all $0<r \leq 2^{k}$ the random formula $\mathbf{\Phi}$ has the following property w.h.p.

There is no set $Q$ of $1 \leq|Q| \leq \chi n$ variables such that the number of clauses containing at least two variables from $Q$ is at least $2|Q|$.

Proof. We use a first moment argument. Let $1 \leq q \leq \chi n$ and let $Q_{0}=\left\{x_{1}, \ldots, x_{q}\right\}$ be a fixed set of size $q$. For any set $Q$ we let $Y(Q)$ be the number of clauses containing at least two variables from $Q$. Moreover, let $X_{q}$ be the number of sets $Q$ of size $q$ such that $Y(Q) \geq 2 q$. Since the distribution $F_{k}(n, m)$ is symmetric with respect to permutations of the variables, we have

$$
\mathrm{E} X_{q} \leq\left(\begin{array}{l}
n \\
q
\end{array}\right) \cdot \mathrm{P}\left[Y\left(Q_{0}\right) \geq 2 q\right] \leq \exp [q(1+\ln (n / q))] \cdot \mathrm{P}\left[Y\left(Q_{0}\right) \geq 2 q\right] .
$$

Furthermore, the probability that a random $k$-clause contains two variables from $Q_{0}$ is at most $\left(\begin{array}{c}k \\ 2\end{array}\right)(q / n)^{2}$ (because for each of the $\left(\begin{array}{l}k \\ 2\end{array}\right)$ pairs of 'slots' in the clauses the probability that both of them are occupied by variables from $Q_{0}$ is at most $\left.(q / n)^{2}\right)$. As $F_{k}(n, m)$ consists of $m$ independent $k$-clauses, $Y\left(Q_{0}\right)$ is stochastically dominated by a binomial random variable $\operatorname{Bin}\left(m,\left(\begin{array}{c}k \\ 2\end{array}\right)(q / n)^{2}\right)$. Consequently, assuming that $q / n \leq \chi$ is sufficiently small, we get

$$
\begin{aligned}
\mathrm{P}\left[Y\left(Q_{0}\right) \geq 2 q\right] & \leq \mathrm{P}\left[\operatorname{Bin}\left(m,\left(\begin{array}{l}
k \\
2
\end{array}\right) q\right) \geq 2 q\right] \\
& \leq \exp \left[-1.9 q \cdot\left[\ln \left(\frac{2 q}{\left(\begin{array}{c}
k \\
2
\end{array}\right)(q / n)^{2} m}\right)-1\right]\right] \quad \text { [by the Chernoff bound (11)] } \\
& \leq \exp \left[-1.9 q \cdot \ln \left(\frac{4}{\mathrm{e} k^{2} r} \cdot \frac{n}{q}\right)\right] \leq \exp \left[-1.9 q \cdot \ln \left(\frac{4}{\mathrm{e} k^{2} 2^{k}} \cdot \frac{n}{q}\right)\right] .
\end{aligned}
$$

Choosing $\chi=\chi(k)$ sufficiently small, we can ensure that $(q / n)^{1 / 4} \leq \chi^{1 / 4} \leq 4 /\left(\mathrm{e} k^{2} 2^{k}\right)$. Plugging this bound into 177, we get

$$
\mathrm{P}\left[Y\left(Q_{0}\right) \geq 2 q\right] \leq \exp [-1.1 q \cdot \ln (n / q)] .
$$

Combining (16) and (18), we get $\mathrm{E} X_{q} \leq \exp [-0.1 q \ln (n / q)]$. In effect, $\mathrm{E} \sum_{1<q<\chi n} X_{q}=O\left(n^{-0.1}\right)$. Hence, Markov's inequality implies that w.h.p. $\sum_{1 \leq q \leq \chi n} X_{q}=0$, in which case (15) holds.

\section{Proof of Theorem B.1}

\section{D.1 Loose variables}

Let $\sigma$ be a satisfying assignment of a $k$-CNF $\Phi$. Remember that a literal $l$ supports a clause $C$ of $\Phi$ if $l$ is the only literal in $C$ that is true under $\sigma$. Moreover, we say that a literal $l$ is 1-loose if it is true under $\sigma$ and supports no clause. In addition, $l$ is 2-loose if $l$ is true under $\sigma$ and each clause that $l$ supports contains a 1-loose literal from $L_{t}$. Thus, any 1-loose literal is 2-loose as well. The key step of the proof is to establish the following.

Proposition D.1 Suppose that $\theta \geq 3 \exp (\rho)(\ln \rho+10) / k$ and $r \leq 2^{k} \ln 2-k$. Let $(\Phi, \sigma)$ be a random pair chosen from the distribution $\mathcal{U}_{k}(n, m)$. Then there are at least $0.999 \theta n$ 2-loose literals in $L_{t}$ w.h.p.

To prove Proposition D.1, we start by estimating the number of 1-loose variables.

Lemma D.2 Suppose that $\theta \geq \exp (\rho) / k$ and $\rho \leq k \ln 2$. Let $(\Phi, \sigma)$ be a random pair chosen from the distribution $\mathcal{P}_{k}^{\prime}(n, m)$. With probability at least $1-\exp \left(-k 2^{2-k} n\right)$ the number of 1 -loose in $L_{t}$ is at least $\theta n \cdot \exp (-\rho) / 2$.

Proof. By LemmaC.3 the number $X$ of 1-loose literals in $L_{t}$ has a binomial distribution with mean

$$
\begin{aligned}
\mathrm{E} X & =\theta n \cdot \mathrm{P}\left[\operatorname{Bin}\left(\frac{k}{n} \cdot\left(\begin{array}{l}
n \\
k
\end{array}\right), \frac{m}{\left(2^{k}-1\right)\left(\begin{array}{l}
n \\
k
\end{array}\right)}\right)=0\right] \\
& =\theta n \cdot\left(1-\frac{m}{\left(2^{k}-1\right)\left(\begin{array}{l}
n \\
k
\end{array}\right)}\right)^{\frac{k}{n} \cdot\left(\begin{array}{l}
n \\
k
\end{array}\right)} \sim \theta n \cdot \exp \left(-\frac{k r}{2^{k}-1}\right)=\theta n \exp \left(-\rho-\rho /\left(2^{k}-1\right)\right) .
\end{aligned}
$$


As $\theta \geq \exp (\rho) / k$ and $\rho \leq k \ln 2$, the Chernoff bound (11) shows that for large enough $k$

$$
\mathrm{P}[X<\theta n \exp (-\rho) / 2] \leq \exp \left[-\frac{\theta n}{8 \exp (\rho)}\right] \leq \exp \left(-k 2^{2-k} n\right)
$$

as desired.

Lemma D.3 Suppose that $\theta \geq 3 \exp (\rho)(\ln \rho+10) / k, \rho \geq \rho_{0}$ with $\rho_{0}$ as in LemmaD.2 and that $k$ is sufficiently large. Let $(\Phi, \sigma)$ be a random pair chosen from the distribution $\mathcal{P}_{k}^{\prime}(n, m)$. Then with probability at least $1-$ $\exp \left(-k 2^{1-k} n\right)$ the number of 2-loose literals in $L_{t}$ is at least $0.999 \theta n$.

Proof. To simplify the notation, we are going to condition on $\sigma$ being the all-true assignment; this is without loss of generality. For each variable $x \in V_{t}$ we let $S_{x}$ be the number of clauses supported by $x$. Moreover, let $S=\sum_{x \in V_{t}} S_{x}$ and let $X$ be the number of variables $x \in V_{t}$ such that $S_{x}=0$. Thus, $X$ equals the number of 1-loose variables.

Let $\mathcal{E}$ be the event that $X \geq \theta n \exp (-\rho) / 2$ and $S \leq 2 \rho \theta n$. Since the number of possible clauses with precisely one positive literal in $L_{t}$ is $\theta n\left(\begin{array}{l}n-1 \\ k-1\end{array}\right), S$ has a binomial distribution $\operatorname{Bin}\left[\theta n\left(\begin{array}{l}n-1 \\ k-1\end{array}\right), m /\left(\left(2^{k}-1\right)\left(\begin{array}{l}n \\ k\end{array}\right)\right)\right]$. Therefore, LemmaD.2 implies that

$$
\begin{aligned}
\mathrm{P}[\neg \mathcal{E}] & \leq \mathrm{P}[X<\theta n \exp (-\rho) / 2]+\mathrm{P}[S>2 \rho \theta n] \\
& \leq \exp \left[-k 2^{2-k} n\right]+\mathrm{P}\left[\operatorname{Bin}\left(\theta n\left(\begin{array}{c}
n-1 \\
k-1
\end{array}\right), \frac{m}{\left(2^{k}-1\right)\left(\begin{array}{l}
n \\
k
\end{array}\right)}\right)>2 \rho \theta n\right] .
\end{aligned}
$$

We have

$$
\theta n\left(\begin{array}{l}
n-1 \\
k-1
\end{array}\right) \cdot \frac{m}{\left(2^{k}-1\right)\left(\begin{array}{l}
n \\
k
\end{array}\right)} \leq \frac{2^{k}}{2^{k}-1} \cdot \rho \theta n .
$$

Hence, combining (19) with the Chernoff bound 11, we obtain for sufficiently large $k$

$$
\mathrm{P}[\neg \mathcal{E}] \leq \exp \left[-k 2^{2-k} n\right]+\exp [-0.99 \rho \theta n] \leq 2 \exp \left[-k 2^{2-k} n\right]
$$

where in the last step we used the assumption that $\rho \geq \rho_{0}$ for a fixed constant $\rho_{0}>0$.

Let us now condition on the event that $S=s$ for some number $s \leq 2 \rho n$, and on the event $\mathcal{E}$. In this conditional distribution for each of the $s$ clauses supported by some variable in $V_{t}$ the $k-1$ negative literals that the clause contains are independently uniformly distributed. Therefore, for each such clause the number of negative literals $\bar{y}$ whose underlying variable $y$ is 1 -loose is binomially distributed $\operatorname{Bin}(k-1, X / n)$. Consequently, the number $T$ of clauses supported by some variable in $V_{t}$ in which no 1-loose variable occurs negatively has a binomial distribution with mean $s \cdot \mathrm{P}[\operatorname{Bin}(k-1, X / n)=0]$. Hence,

$$
\begin{aligned}
\mathrm{E}[T \mid \mathcal{E}] & \leq 2 \rho \theta n \cdot \mathrm{P}[\operatorname{Bin}(k-1, \theta \exp (-\rho) / 2)=0] \\
& =2 \rho \theta n \cdot(1-\theta \exp (-\rho) / 2)^{k-1} \leq 2 \rho \theta n \exp (-\theta \exp (-\rho) k / 3) \leq 2 \exp (-10) \theta n .
\end{aligned}
$$

Thus, the Chernoff bound (11) implies that for $k \geq k_{0}$ large enough

$$
\mathrm{P}[T>0.001 \theta n \mid \mathcal{E}] \leq \exp (-0.001 \theta n) \leq \exp \left[-k 2^{2-k} n\right] .
$$

Finally, the assertion follows from (20) and (21).

Proof.[Proposition D.1] Let $\mathcal{E}$ be the event that a pair $(F, \sigma) \in \Lambda_{n, m}$ has at least $0.999 \theta n$ 2-loose literals. Lemma D.3 shows that

$$
\mathrm{P}_{\mathcal{P}_{k}^{\prime}(n, m)}[\mathcal{E}] \geq 1-\exp \left(-k 2^{1-k} n\right) \geq 1-\exp \left(-k r n / 4^{k}\right) .
$$

Moreover, Corollary C.2 and (22) imply that $\mathrm{P}_{\mathcal{U}_{k}(n, m)}[\mathcal{E}]=1-o(1)$ as desired.

Proof.[Theorem B.1, part 1] By Fact4.1] it suffices to prove the desired statement for the experiment U1-U4. Thus, let $(\Phi, \sigma)$ be a pair chosen from the distribution $\mathcal{U}_{k}(n, m)$. Without loss of generality we may condition on $\sigma$ being the all-true assignment. Let $\mathcal{L}$ be the set of all tame variables that are 2-loose. Then by Propositions C.4 and D.1 we have $\mathcal{L} \geq(0.999-o(1)) \theta n \geq 0.99 \theta n$ w.h.p. Assuming that this is the case, we are going to show that if $x \in \mathcal{L}$, then there is a satisfying assignment $\tau$ such that $\tau(x) \neq \sigma(x)$ and $\operatorname{dist}(\tau, \sigma) \leq \ln (n)$. 
Thus, fix a variable $x \in \mathcal{L}$. If $x$ is 1-loose, then we can just set $\tau(x)=1-\sigma(x)=0$ and $\tau(y)=\sigma(y)=1$ for all $y \neq x$ to obtain a satisfying assignment with $\operatorname{dist}(\tau, \sigma)=1$, because $x$ does not support any clauses. Hence, assume that $x$ is 2 -loose but not 1 -loose. Let $\mathcal{C}$ be the set of all clauses supported by $x$ in $(\Phi, \sigma)$. Any clause $C \in \mathcal{C}$ contains a negative occurrence of a 1-loose variable $x_{C} \in V_{t}$ in $C$ (by the very definition of 2-loose). Define $\tau(x)=0, \tau\left(x_{C}\right)=0$ for all $C \in \mathcal{C}$, and $\tau(y)=\sigma(y)=1$ for all other variables $y$.

We claim that $\tau$ is a satisfying assignment. To see this, assume for contradiction that there is a clause $U$ that is unsatisfied under $\tau$. Then $U$ contains a variable from $\{x\} \cup\left\{x_{C}: C \in \mathcal{C}\right\}$ positively, while none of these variables occurs negatively in $U$. Hence, $U \notin \mathcal{C}$. Moreover, since the variables $x_{C}, C \in \mathcal{C}$, do not support any clauses, $U$ indeed contains two variables from the set $\{x\} \cup\left\{x_{C}: C \in \mathcal{C}\right\}$ positively. There are two possible cases.

Case 1: $x$ occurs in $U$. Let $C \in \mathcal{C}$ such that $x_{C}$ occurs in $U$ as well. Then the factor graph contains the cycle $x, C, x_{C}, U, x$, in contradiction to our assumption that $x$ is tame.

Case 2: $x$ does not occur in $U$. There exist $C_{1}, C_{2} \in \mathcal{C}$ such that $x_{C_{1}}, x_{C_{2}}$ occur in $C$. Hence, the factor graph contains the cycle $x, C_{1}, x_{C_{1}}, C, x_{C_{2}}, C_{2}, x$, once more in contradiction to the assumption that $x$ is tame.

Hence, there is no clause $U$ that is unsatisfied under $\tau$. Finally, since all the variable $x_{C}$ with $C \in \mathcal{C}$ have distance two from $x$ in the factor graph, and as $x$ is tame, we have $\operatorname{dist}(\sigma, \tau) \leq \ln n$.

\section{D.2 Rigid variables}

The proof of the second part of Theorem B.1 follows the outline given in Section 6 Recall the function $\varphi$ from (12).

Proposition D.4 Suppose that $k \geq 6$ and $0<r \leq 2^{k} \ln 2-k$. Let $\mu=\rho \cdot 2^{k} /\left(2^{k}-1\right)$ and $\zeta=(1+\mu+$ $\left.\mu^{2} / 2\right) / \exp (\mu)$, and assume that $2^{\bar{k}} \theta \zeta \varphi(1)>\rho$. Then w.h.p. in a random pair $(\Phi, \sigma)$ chosen from the distribution $\mathcal{U}_{k}(n, m)$ no more than $2 \zeta \theta$ n literals in $L_{t}$ support fewer than three clauses.

Proof. Let $S$ be the number of literals $l \in L_{t}$ that support fewer than three clauses. We are going to show that

$$
\mathrm{P}_{\mathcal{P}_{k}(n, m)}[S>2 \zeta \theta n] \leq \exp \left(-k r n / 4^{k}\right)
$$

Then Corollary C.2 implies the assertion.

In the distribution $\mathcal{P}_{k}^{\prime}(n, m)$ the random variable $S$ is binomially distributed with mean $(1+o(1)) \theta \zeta n$ by the second part of Lemma C.3. Hence, the Chernoff bound (11) shows that

$$
\mathrm{P}_{\mathcal{P}_{k}(n, m)}[S>2 \zeta n] \leq \exp (-(1+o(1)) \theta \zeta \varphi(1) n) .
$$

By the assumptions on $\mu$ and $\theta$ we have $\theta \zeta \varphi(1)>\rho / 2^{k}$; hence, 23) follows from 24).

Remember that a set $\mathcal{S} \subset L_{t}$ of literals $t$-self-contained if each literal $l \in \mathcal{S}$ supports at least two clauses that contain literals from $\left\{x_{1}, \bar{x}_{1}, \ldots, x_{t}, \bar{x}_{t}\right\} \cup \mathcal{S} \cup \overline{\mathcal{S}}$ only, where $\overline{\mathcal{S}}$ is the set of all negations of literals in $\mathcal{S}$.

Proposition D.5 For any $k \geq 3$ there is a number $\chi=\chi(k)>0$ such that for any $0<r \leq 2^{k} \ln 2-k$ the following is true. Let $(\Phi, \sigma)$ be a random pair chosen from the distribution $\mathcal{U}_{k}(n, m)$. Then w.h.p. for any t-self-contained set $\mathcal{S}$ all variables $x \in \mathcal{S} \cup \overline{\mathcal{S}}$ are $\chi n$-rigid.

Proof. Let $(\Phi, \sigma)$ be a random pair chosen from the distribution $\mathcal{U}_{k}(n, m)$. Without loss of generality we may condition on $\sigma$ being the all-true assignment. By Lemma C.5 there is a number $\chi=\chi(k)>0$ such that (15) is satisfied w.h.p., and we are going to assume that this is the case.

Let $\mathcal{S}$ be a self-contained set. Suppose that $\tau$ is a satisfying assignment such that the set $Q$ of all variables $x \in \mathcal{S} \cup \overline{\mathcal{S}}$ such that $\tau(x) \neq \sigma(x)$ is non-empty. For each variable $x \in Q$ there are two clauses $C_{1}(x), C_{2}(x)$ that are supported by $x$ in $\sigma$ and that consist of literals from $\mathcal{S} \cup \overline{\mathcal{S}}$ only (because $\mathcal{S}$ is self-contained). Since $\tau$ is satisfying and $\tau(x) \neq \sigma(x)$, both $C_{1}(x)$ and $C_{2}(x)$ contain another variable from $Q$. Hence, there are at least $2|Q|$ clauses that contain at least two variables from $Q$. Thus, 15 implies that $|Q|>\chi n$, and consequently $\operatorname{dist}(\sigma, \tau) \geq|Q|>\chi n$. 
Proposition D.6 Suppose that $k \geq 4$ and $0<r \leq 2^{k} \ln 2-k$, and that $0 \leq \theta \leq 1$. Set

$$
\mu=\frac{\rho 2^{k}}{2^{k}-1}, \zeta=\frac{1+\mu+\mu^{2} / 2}{\exp (\mu)}, \lambda=1-(1-3 \theta \zeta)^{k-1}, \gamma=\frac{\mu \cdot(\exp (\lambda \mu)-1-\lambda \mu)}{(1-\zeta) \exp (\mu)}
$$

and let $h(x)=-x \ln x-(1-x) \ln (1-x)$. If $\zeta<1 / 3$ and

$$
\theta(\zeta \ln (\gamma)+h(\zeta))+\rho / 2^{k}<0
$$

then a random pair $(\Phi, \sigma)$ chosen from the distribution $\mathcal{U}_{k}(n, m)$ has one of the following properties w.h.p.

a. More than $2 \theta \zeta n$ literals in $L_{t}$ that are true under $\sigma$ literals support fewer than three clauses.

b. There is a t-self-contained set of size $(1-3 \zeta) \theta n$.

Proof. Let $p=m /\left(\left(2^{k}-1\right)\left(\begin{array}{l}n \\ k\end{array}\right)\right)$. Let $(\Phi, \sigma)$ be chosen from the distribution $\mathcal{P}_{k}(n, m)$. We may condition on $\sigma$ being the all-true assignment, and on the event that at most $2 \zeta \theta n$ literals amongst $x_{t+1}, \bar{x}_{t+1}, \ldots, x_{n}, \bar{x}_{n}$ that are true under $\sigma$ support fewer than three clauses (as otherwise a. occurs). In fact, fix a set $Z$ of $2 \zeta \theta n$ variables and condition on the event $\mathcal{E}$ that all variables that support at most two clauses lie in $Z$. For any variable $x \notin Z$ we let $S_{x}$ be the number of clauses supported by $x$. Then the first part of Lemma C.3 implies that $S_{x}$ has a binomial distribution $\operatorname{Bin}\left(k\left(\begin{array}{l}n-1 \\ k-1\end{array}\right), p\right)$ conditioned on the outcome being at least three. As a consequence, for any $j \geq 3$

$$
\mathrm{P}\left[S_{x}=j \mid \mathcal{E}\right]=\frac{\mathrm{P}\left[\operatorname{Bin}\left(k\left(\begin{array}{l}
n-1 \\
k-1
\end{array}\right), p\right)=j\right]}{\mathrm{P}\left[\operatorname{Bin}\left(k\left(\begin{array}{l}
n-1 \\
k-1
\end{array}\right), p\right)<3\right]} \leq \frac{(1+o(1)) \mu^{j}}{j ! \exp (\mu)(1-\zeta)}+O(\exp (-j / \mu) / n) .
$$

Let $X \subset\left\{x_{t+1}, \ldots, x_{n}\right\} \backslash Z$ be a set of $\zeta \theta n$ variables. For each $x \in X$ we let $T_{x}$ be the number of clauses supported by $x$ in which a variable from $X \cup Z$ occurs negatively. In a random clause supported by $x$ the variables underlying the $k-1$ negative literals in that clause are distributed uniformly over $V$. Therefore, given $\mathcal{E}$ the probability that such a clause contains at least one variable from $X \cup Z$ is

$$
1-(1-|X \cup Z| / n)^{k-1}+o(1)=1-(1-3 \theta \zeta)^{k-1}+o(1) \sim \lambda
$$

Hence, if we condition on both $\mathcal{E}$ and $S_{x}=j$, then the probability that $T_{x} \geq S_{x}-1$ equals $j \cdot(\lambda+o(1))^{j-1}$. Thus, letting $\gamma=\mu \cdot(\exp (\lambda \mu)-1-\lambda \mu) /(1-\zeta) \exp (\mu)$, we obtain from (26)

$$
\begin{aligned}
\mathrm{P}\left[T_{x} \geq S_{x}-1 \mid \mathcal{E}\right] & =\sum_{j \geq 3} \mathrm{P}\left[T_{x} \geq S_{x}-1 \mid \mathcal{E} \text { and } S_{x}=j\right] \cdot \mathrm{P}\left[S_{x}=j \mid \mathcal{E}\right] \\
& \leq(1+o(1)) \sum_{j \geq 3} \frac{j \lambda^{j-1} \mu^{j}}{j ! \exp (\mu)(1-\zeta)} \sim \gamma .
\end{aligned}
$$

Given that $\mathcal{E}$ occurs the events $T_{x} \geq S_{x}-1$ are mutually independent for all $x \in X$. Therefore,

$$
\mathrm{P}\left[\forall x \in X: T_{x} \geq S_{x}-1 \mid \mathcal{E}\right] \leq(\gamma+o(1))^{\theta \zeta n} .
$$

If b. does not occur, then there is a set $X \subset V \backslash Z$ of size $\zeta n$ such that $T_{x} \geq S_{x}-1$ for all $x \in X$. Hence, by the union bound the probability that $b$. does not occur is at most

$$
\begin{aligned}
\mathrm{P}[\exists X \subset V \backslash Z & \left.,|X|=\zeta n: \forall x \in X: T_{x} \geq S_{x}-1 \mid \mathcal{E}\right] \leq \sum_{X \subset V \backslash Z,|X|=\theta \zeta n} \mathrm{P}\left[\forall x \in X: T_{x} \geq S_{x}-1 \mid \mathcal{E}\right] \\
& \leq\left(\begin{array}{c}
(1-2 \zeta) \theta n \\
\theta \zeta n
\end{array}\right) \cdot(\gamma+o(1))^{\theta \zeta n} \leq \exp [(1-2 \zeta) h(\zeta /(1-2 \zeta)) \cdot n] \cdot(\gamma+o(1))^{\zeta n} \\
& \leq \exp [\theta n \cdot((1-2 \zeta) h(\zeta /(1-2 \zeta))+\zeta \cdot \ln \gamma+o(1))]<\exp \left(-\rho n / 2^{k}\right) \quad[\text { by (25)] }
\end{aligned}
$$

as desired. Finally, the assertion follows directly from Corollary C.2

Proof.[Theorem B.1, part 2] Suppose that $1 / k \leq \theta \leq \exp (\rho) /\left(\rho^{3} k\right)$. The goal is to verify (25). Since $h(\zeta) \leq \zeta(1-\ln \zeta)$, proving (25) reduces to showing $\theta \zeta[\ln \gamma+1-\ln \zeta]<-\rho / 2^{k}$, i.e.,

$$
\theta \zeta \ln (\mathrm{e} \gamma / \zeta)<-\rho / 2^{k} .
$$


Plugging in the definitions of $\gamma$ and $\zeta$, we see that

$$
\ln \left(\frac{\mathrm{e} \gamma}{\zeta}\right)=\ln \left[\frac{\mathrm{e} \mu(\exp (\lambda \mu)-\lambda \mu-1)}{(1-\zeta)\left(1+\mu+\mu^{2} / 2\right)}\right] \leq \ln \left(\frac{5 \mathrm{e}[\exp (\lambda \mu)-\lambda \mu-1]}{\mu}\right) \quad \text { [for } \mu \text { not too small]. }
$$

Since $3 \theta \zeta \leq 4 /(k \rho)$ for $\rho \geq \rho_{0}$ sufficiently large, we have $\lambda=1-(1-3 \theta \zeta)^{k-1} \leq 4 k \theta \zeta$. Hence,

$$
\lambda \mu \leq 4 k \mu \theta \zeta \leq \frac{4 \mu^{2}}{\exp (\mu)} \cdot \frac{\exp (\mu)}{\mu^{3}} \leq 4 / \mu
$$

Therefore, we obtain for $\mu \geq \rho \geq \rho_{0}$ large

$$
\ln \left(\frac{\mathrm{e} \gamma}{\zeta}\right) \leq \ln \left(\frac{4 \mathrm{e}(\lambda \mu)^{2}}{\mu}\right) \leq \ln \left(64 \mathrm{e} / \mu^{3}\right) \leq-1
$$

As $\theta \zeta \geq \zeta / k \geq \frac{1}{2} \mu^{2} \exp (-\mu) \geq \frac{1}{3} \rho^{2} / 2^{k}$ for $k \geq k_{0}$ and $\rho \geq \rho_{0}$ not too small, we thus obtain 27.

\section{D.3 Forced variables}

Let $(\Phi, \sigma)$ be a formula/assignment pair. A clause $C$ forces a variable $x \in V_{t}$ if $C$ contains $k-1$ literals from $\left\{x_{1}, \bar{x}_{1}, \ldots, x_{t}, \bar{x}_{t}\right\}$, none of which satisfies $C$ under $\sigma$, and either the literal $x$ or $\bar{x}$, which does.

Lemma D.7 Suppose that $\rho \geq \rho_{0}, k \geq k_{0}$, and $k \theta \sim \ln (\rho)-10$. Then w.h.p. in a pair $(\Phi, \sigma)$ chosen from the distribution $\mathcal{U}_{k}(n, m)$ at least $0.9910 n$ variables in $V_{t}$ are forced.

Proof. Let $\mathcal{F}$ be the event that at least $0.991 \theta n$ variables in $V_{t}$ are forced. We are going to show that

$$
\mathrm{P}_{\mathcal{P}_{k}^{\prime}(n, m)}[\mathcal{F}] \geq 1-\exp \left(-1.1 \rho / 2^{k}\right)
$$

so that the assertion follows from Corollary C.2

Thus, let $\left(\Phi^{\prime}, \sigma^{\prime}\right)$ be a pair chosen from the distribution $\mathcal{P}_{k}^{\prime}(n, m)$. We may assume without loss of generality that $\sigma^{\prime}$ is the all-true assignment. For each variable $x \in V_{t}$ the number of clauses that $x$ supports has a binomial distribution with mean $\mu=\rho \cdot 2^{k} /\left(2^{k}-1\right)$. Furthermore, if $C$ is a random clauses supported by $x$, then $C$ contains $k-1$ random negative literals; the probability that all of these are in $V \backslash V_{t}$ equals $(1-\theta+o(1))^{k-1}$. Hence, the number $F_{x}$ of forcing clauses for $x$ is binomially distributed with mean

$$
\begin{aligned}
\mathrm{E}\left[F_{x}\right] & =\mu(1-\theta+o(1))^{k-1} \geq \rho(1-\theta)^{k-1} \\
& \geq \rho \exp \left[-\left(\theta+\theta^{2}\right)(k-1)\right] \geq \rho \exp \left[-\theta k-\theta^{2} k\right] \geq \exp (5)
\end{aligned}
$$

Therefore, for any $x \in V_{t}$ we have $\mathrm{P}\left[F_{x}=0\right] \leq \exp (-\exp (5))$, and the events $\left(\left\{F_{x}=0\right\}\right)_{x \in V_{t}}$ are mutually independent. Hence, the number $Z$ of variables $x \in V_{t}$ with $F_{x}=0$ is binomially distributed with mean $\exp (-\exp (-5)) \theta n$, and thus

$$
\mathrm{P}[Z \geq 0.009 \theta n] \leq \exp (-0.009 \theta n) \leq \exp \left(-1.1 \rho / 2^{k}\right)
$$

by Chernoff bounds. This proves (28).

Proof.[Theorem B.1 part 3] To complete the proof of Theorem B.1, part 3, we need to deal with general values $1 / n \ll \theta \leq \theta_{0}=(\ln (\rho)-10) / k$. Let $t=(1-\theta) n$ and $t_{0}=\left(1-\theta_{0}\right) n$. To obtain a pair $\left(\Phi_{t}, \sigma_{t}\right)$ from the distribution $\mathbf{U 1 - U 4}$, one can proceed as follows. First, choose a pair $\left(\Phi_{t_{0}}, \sigma_{t_{0}}\right)$ from the distribution U1-U4 with $t_{0}$ variables decimated. Then, assign the variables in $x \in V_{t_{0}} \backslash V_{t}$ with the truth values $\sigma_{t_{0}}(x)$, simplify the formula, and let $\sigma_{t}(y)=\sigma_{t_{0}}(y)$ for all $y \in V_{t}$. We are going to use this experiment to analyze the number of forced variables in $\left(\Phi_{t}, \sigma_{t}\right)$.

The above experiment shows that any variable $x \in V_{t}$ that is forced in $\left(\Phi_{t_{0}}, \sigma_{0}\right)$ remains forced in $\left(\Phi_{t}, \sigma_{t}\right)$. Let $\mathcal{F}$ be the set of forced variables in $\left(\Phi_{t_{0}}, \sigma_{0}\right)$. Given that $|\mathcal{F}|=j$, the set $\mathcal{F}$ is a uniformly random subset of $V_{t_{0}}$. Hence, if we condition on the event that $|\mathcal{F}| \geq 0.991 \theta_{0} n$, then $\left|\mathcal{F} \cap V_{t}\right|$ has a hypergeometric distribution with mean at least $0.991 \theta n$. Therefore, by Chebyshev's inequality, we have $\left|\mathcal{F} \cap V_{t}\right| \geq(0.991 \theta-o(1)) n \geq 0.99 \theta n$ w.h.p. (here we use that $\theta n \gg 1$ ). Thus, the theorem follows from Lemma D.7 


\section{E Proof of Theorem B.2}

\section{E.1 Shattering}

In this section we prove the first part of Theorem B.2. Consider a pair $(\Phi, \sigma)$ chosen from the planted model $\mathcal{P}_{k}(n, m)$. Let $\Phi_{t, \sigma}$ denote the formula obtained from $\Phi$ by substituting the values $\sigma\left(x_{1}\right), \ldots, \sigma\left(x_{t}\right)$ for the first $t$ variables. Without loss of generality, we may assume that $\sigma=\mathbf{1}$ is the all-true assignment. The main step of the proof is the summarized in the following proposition.

Proposition E.1 Let $k \geq 6$ and $r>0$ be fixed. Moreover, let $0<\theta \leq 1$ and let

$$
\psi:(0,1) \rightarrow \mathbf{R}, \quad \alpha \mapsto-\alpha \theta \ln \alpha-(1-\alpha) \theta \ln (1-\alpha)+r \ln \left(1-\frac{1-(1-\alpha \theta)^{k}}{2^{k}-1}\right) .
$$

Suppose that there is a number $a \in(0,1)$ such that

$$
\psi(a)+\rho / 2^{k}<0 \quad \text { and } \sup _{0<\alpha<a} \psi(\alpha)<\theta \ln 2+2^{k} \rho \ln \left(1-2^{-k}\right) / k-\rho / 2^{k} .
$$

Then there is $\varepsilon=\varepsilon(k, \rho)$ such that for $\Phi_{t}$ generated by the experiment $\mathbf{U 1}-\mathbf{U} \mathbf{4}$, the set $\mathcal{S}\left(\Phi_{t}\right)$ is $(a-\varepsilon, a+\varepsilon)$ shattered.

We will first show how Proposition E.1 implies the first part of Theorem B.2. The proof of Proposition E.1 appears at the end of this section. To derive the first part of Theorem B.2 from Proposition E.1 we need to verify (29).

Lemma E.2 Assume that $0 \leq \theta \leq \exp (\rho-2) /(\rho k)$. Let $a=\exp (2-\rho)$. Then $\psi(a)<-a \theta / 2$.

Proof. We have

$$
\begin{aligned}
\psi(a) & \leq a \theta(1-\ln a)-\frac{\rho}{k}\left(1-(1-a \theta)^{k}\right) \leq a \theta(1-\ln a)-\frac{\rho}{k}(1-\exp (-a k \theta)) \\
& \leq a \theta(1-\ln a)-\frac{\rho}{k}\left(a k \theta-(a k \theta)^{2} / 2\right)=a \theta[1-\ln a-\rho(1-a k \theta / 2)],
\end{aligned}
$$

where we used $\exp (-z) \leq 1-z+z^{2} / 2$ for $z \geq 0$. Since $k \theta \rho \leq \exp (\rho-2)$ by assumption, our choice of $a$ implies that $\psi(a) \leq a \theta[1-\ln a-\rho+a \exp (\rho-2) / 2]=-a \theta / 2$, as claimed.

Lemma E.3 Assume that $0 \leq \theta \leq \exp (\rho-2) /(\rho k)$. Let $a=\exp (2-\rho)$. Then $\sup _{\alpha<a} \psi(\alpha) \leq \frac{3}{2 \mathrm{e}^{2} k \rho}$.

Proof. Let $0 \leq \alpha<a$. We have

$$
\psi(\alpha) \leq \theta(\alpha-\alpha \ln \alpha-\alpha \rho(1-\alpha k \theta / 2))
$$

Let $\psi_{1}(\alpha)$ be the expression on the r.h.s. Then

$$
\frac{d}{d \alpha} \psi_{1}(\alpha)=\theta[-\ln \alpha-\rho+\alpha k \theta], \quad \frac{d^{2}}{d \alpha^{2}} \psi_{1}(\alpha)=\theta[k \theta-1 / \alpha] .
$$

Thus, our assumption on $\theta$ implies that $\frac{d^{2}}{d \alpha^{2}} \psi_{1}(\alpha)<0$ for all $0<\alpha<a$, and therefore $\psi_{1}$ has a unique local maximum in the interval $(0, \alpha)$. To pinpoint this maximum, note that for $\alpha_{0}=\exp (-\rho)$ the first derivative $\frac{d}{d \alpha} \psi_{1}\left(\alpha_{0}\right)$ is positive. Moreover, at $\alpha_{1}=\exp (1-\rho)$ we have $\frac{d}{d \alpha} \psi_{1}\left(\alpha_{1}\right)<0$. Hence, the unique local maximum of $\psi_{1}$ lies in the interval $\left(\alpha_{0}, \alpha_{1}\right)$. To study the maximum value, consider the function $\psi_{2}: \alpha \mapsto \alpha-\alpha \ln \alpha-\alpha \rho$. Its derivative is $d / d \alpha \psi_{2}(\alpha)=\rho-\ln \alpha$, so that the maximum of this function occurs at $\alpha_{0}$. Furthermore, the quadratic term $\alpha \mapsto \alpha^{2} k / 2$ is monotonically increasing in $\alpha$. Therefore,

$$
\sup _{0<\alpha<a} \psi(\alpha) \leq \sup _{0<\alpha<a} \psi_{1}(\alpha)=\sup _{\alpha_{0}<\alpha<\alpha_{1}} \psi_{1}(\alpha) \leq \theta\left(\psi_{2}\left(\alpha_{0}\right)+\alpha_{1}^{2} k / 2\right)=3 \theta \exp (-\rho) / 2 .
$$

Finally, the assertion follows from the assumed bound on $\theta$. 
Proof.[Theorem B.2, part 2] Assume that $\rho \leq k \ln 2-\ln k$ and

$$
\frac{\rho}{k \ln 2}\left(1+\rho^{-2}+2^{2-k}\right) \leq \theta \leq \exp (\rho-2) /(\rho k)
$$

Let $a=\exp (2-\rho)$. LemmaE.2 shows that

$$
\psi(a)+\rho / 2^{k} \leq \rho / 2^{k}-\exp (2-\rho) \theta / 2 \leq \rho / 2^{k}-\frac{\exp (2-\rho) \rho}{k \ln 2}=\frac{\rho}{2^{k}}\left(1-\frac{2^{k} \exp (2-\rho)}{k \ln 2}\right) .
$$

Since $\rho \leq k \ln 2-\ln k$, the r.h.s. is negative. By LemmaE.3 we have

$$
\begin{aligned}
\theta \ln 2+\frac{2^{k} \rho}{k} \ln \left(1-2^{-k}\right)-\rho / 2^{k} & \geq \theta \ln 2-\frac{\rho}{k}-\rho / 2^{k-1} \\
& \geq \frac{1}{k \rho}+2^{2-k} \rho \ln 2-\rho / 2^{k-1} \geq \frac{1}{k \rho}>\sup _{\alpha<a} \psi(\alpha) .
\end{aligned}
$$

Thus, the assertion follows from Proposition E.1

\section{E.1.1 Proof of Proposition E.1.}

In the rest of this section we keep the notation and the assumptions from Proposition E.1. Let

$$
b=\theta \ln 2+2^{k} \rho \ln \left(1-2^{-k}\right) / k-\rho / 2^{k} .
$$

Lemma E.4 There exist numbers $\xi>0,0<a_{1}<a_{2}<1$ such that a pair $(\Phi, \sigma)$ chosen from the distribution $\mathcal{P}_{k}(n, m)$ has the following two properties with probability at least $1-\exp \left(-\left(\xi+\rho / 2^{k}\right) n\right)$.

1. $\Phi_{t, \sigma}$ does not have a satisfying assignment $\tau$ with $a_{1} n<\operatorname{dist}(\sigma, \tau)<a_{2} n$.

2. $\left|\left\{\tau \in \mathcal{S}\left(\Phi_{t, \sigma}\right): \operatorname{dist}(\sigma, \tau)<a_{2} n\right\}\right| \leq \exp ((b-\xi) n)$.

Proof. For $\alpha>0$ we let $X_{\alpha}=\left|\left\{\tau \in \mathcal{S}_{t, \sigma}(F): \operatorname{dist}(\sigma, \tau)=\alpha \theta n\right\}\right|$. Note that

$$
\mathrm{E} X_{\alpha} \leq\left(\begin{array}{c}
\theta n \\
\alpha \theta n
\end{array}\right)\left(1-\frac{1-(1-\alpha \theta)^{k}}{2^{k}-1}\right)^{m}
$$

Taking logarithms and bounding the binomial coefficient via Stirling's formula, we obtain

$$
\frac{\ln \mathrm{E} X_{\alpha}}{n} \leq \psi(\alpha)
$$

Let $a \in(0,1)$ be such that $\psi(a)+\rho / 2^{k}<0$ (cf. (29)). As $\psi$ is continuous there exist $0<a_{1}<a<a_{2}<1$ and $\xi_{1}>0$ such that

$$
\sup _{a_{1} \leq \alpha \leq a_{2}} \psi(\alpha)<-\rho / 2^{k}-2 \xi_{1} .
$$

Combining (32) and (33), we conclude that $\mathrm{E} X_{\alpha} \leq \exp \left[-n\left(\rho / 2^{k}+2 \xi_{0}\right)\right]$ for all $a_{1} \leq \alpha \leq a_{2}$. Summing over integers $a_{1} n \leq j \leq a_{2} n$, we see that for large $n$

$$
\sum_{a_{1} n \leq j \leq a_{2} n} \operatorname{EX}_{j / n, \xi_{1}} \leq n \exp \left[-n\left(\rho / 2^{k}+2 \xi_{0}\right)\right] \leq \exp \left[-n\left(\rho / 2^{k}+\xi_{0}\right)\right] .
$$

Hence, by Markov's inequality the probability that there is a satisfying assignment $\tau$ that coincides with $\sigma$ on the first $t$ variables such that $a_{1} n \leq \operatorname{dist}(\sigma, \tau) \leq a_{2} n$ is bounded by $\exp \left(-n\left(\rho / 2^{k}+\xi_{0}\right)\right)$. This proves the first assertion.

Since we are assuming that $\sup _{0<\alpha<a} \psi(\alpha)<b-\rho / 2^{k}$, and as 33 shows that $\psi(\alpha)<-\rho / 2^{k}-2 \xi_{1}<$ $b-\rho / 2^{k}-2 \xi_{1}$ for all $a \leq \alpha<a_{2}$, there is a number $\xi_{2}>0$ such that

$$
\sup _{0<\alpha \leq a_{2}} \psi(\alpha)<b-\rho / 2^{k}-3 \xi_{2}
$$


Hence, 32 implies that

$$
\mathrm{E} X_{\alpha} \leq \exp (n \psi(\alpha)) \leq \exp \left(n\left(b-\rho / 2^{k}-3 \xi_{2}\right)\right) \quad \text { for all } 0<\alpha \leq a_{2}
$$

Taking the sum over integers $0 \leq j \leq a_{2} n$, we get for large enough $n$

$$
\sum_{0 \leq j \leq a_{2} n} \mathrm{E} X_{j / n} \leq n \exp \left(n\left(b-\rho / 2^{k}-3 \xi_{2}\right)\right) \leq \exp \left(n\left(b-\rho / 2^{k}-2 \xi_{2}\right)\right) .
$$

That is, the expected number of assignments $\tau \in \mathcal{S}\left(\Phi_{t, \sigma}\right)$ such that $\operatorname{dist}(\sigma, \tau) \leq a_{2} n$ is bounded by $\exp (n(b-$ $\left.\left.\rho / 2^{k}-2 \xi_{2}\right)\right)$. Hence, Markov's inequality entails that with probability at least $1-\exp \left(-n\left(\rho / 2^{k}+\xi_{2}\right)\right)$ there are at $\operatorname{most} \exp \left(n\left(b-\xi_{2}\right)\right)$ such satisfying assignments $\tau$. This proves the second assertion.

Corollary E.5 There exist numbers $\xi>0,0<a_{1}<a_{2}<1$ such that a pair $(\Phi, \sigma)$ chosen from the distribution $\mathcal{U}_{k}(n, m)$ enjoys the two properties stated in Lemma E.4 with probability at least $1-\exp (-\xi n)$.

Proof. This follows directly from Lemma E.4 and Corollary 4.2 .

Proof.[PropositionE.1] Let $\xi, a_{1}, a_{2}$ be the numbers provided by Corollary E.5] and let $(\Phi, \sigma)$ be a pair chosen from the distribution $\mathcal{U}_{k}(n, m)$. With each assignment $\tau \in \mathcal{S}\left(\Phi_{t, \sigma}\right)$ we associate a set

$$
\mathcal{C}(\tau)=\left\{\chi \in \mathcal{S}\left(\Phi_{t, \sigma}\right): \operatorname{dist}(\chi, \tau) \leq a_{1} n\right\}
$$

Moreover, we call $\tau \in \mathcal{S}\left(\Phi_{t, \sigma}\right)$ good if $|\mathcal{C}(\tau)| \leq \exp ((b-\xi) n)$ and there is no $\chi \in \mathcal{S}\left(\Phi_{t, \sigma}\right)$ such that $a_{1} n \leq$ $\operatorname{dist}(\chi, \tau) \leq a_{2} n$. Let $\mathcal{S}_{\text {good }}$ be the set of all good $\tau \in \mathcal{S}\left(\Phi_{t, \sigma}\right)$ and $\mathcal{S}_{\text {bad }}=\mathcal{S}\left(\Phi_{t, \sigma}\right) \backslash \mathcal{S}_{\text {good }}$. Corollary E.5 and our choice of $b$ ensure that $F$ has the following two properties w.h.p.:

$$
\begin{aligned}
\left|\mathcal{S}\left(\Phi_{t, \sigma}\right)\right| & \geq 2^{t} \exp (b n) \\
\left|\mathcal{S}_{\text {good }}\right| & \geq(1-\exp (-\xi n)) \cdot\left|\mathcal{S}\left(\Phi_{t, \sigma}\right)\right| .
\end{aligned}
$$

Assuming that (34) and 35) hold and that $n$ is sufficiently large, we are going to construct a decomposition of $\mathcal{S}\left(\Phi_{t, \sigma}\right)$ into subsets as required by SH1-SH2. To this end, choose some $\sigma_{1} \in \mathcal{S}_{\text {good }}$. Having defined $\sigma_{1}, \ldots, \sigma_{l}$, we choose an arbitrary $\sigma_{l+1} \in \mathcal{S}_{\text {good }} \backslash \bigcup_{j=1}^{l} \mathcal{C}\left(\sigma_{j}\right)$, unless this set is empty, in which case we stop. Let $\sigma_{1}, \ldots, \sigma_{N}$ be the resulting sequence and define

$$
R_{l}=\mathcal{C}\left(\sigma_{l}\right) \backslash \bigcup_{j=1}^{l-1} \mathcal{C}\left(\sigma_{j}\right) \quad \text { for } 1 \leq l \leq N, \quad \text { and } R_{0}=\mathcal{S}\left(\Phi_{t, \sigma}\right) \backslash \bigcup_{l=1}^{N} R_{l}
$$

Then $\mathcal{S}\left(\Phi_{t, \sigma}\right)=R_{0} \cup \cdots \cup R_{N}$. (Observe that possibly $R_{0}=\emptyset$ while $R_{l} \neq \emptyset$ for all $1 \leq l \leq N$ as $\sigma_{l} \in R_{l}$.) Furthermore, for each $1 \leq l \leq N$ we have $R_{l} \subset \mathcal{C}\left(\sigma_{l}\right)$ and thus

$$
\begin{aligned}
\left|R_{l}\right| & \leq\left|\mathcal{C}\left(\sigma_{l}\right)\right| \leq \exp ((b-\xi) n) & \text { [because } \sigma_{l} \text { is good] } \\
& \leq\left|\mathcal{S}\left(\Phi_{t, \sigma}\right)\right| \cdot \exp (-\xi n) & \text { [by (34)] }
\end{aligned}
$$

Furthermore, as $R_{0} \subset \mathcal{S}_{\text {bad }}, 35$ implies

$$
\left.\left|R_{0}\right| \leq\left|\mathcal{S}_{b a d}\right| \leq \exp (-\xi n)\right) \cdot\left|\mathcal{S}\left(\Phi_{t, \sigma}\right)\right|
$$

Combining (36) and (37) we see that the decomposition $R_{0}, \ldots, R_{N}$ satisfies SH1. Furthermore, SH2 is satisfied by construction.

\section{E.2 Condensation}

Here we prove the second part of Theorem B.2 The following proposition reduces that task to a problem in calculus. 
Proposition E.6 Let $k \geq 3$ and $r>0$ be fixed. Let $0<\theta \leq 1$ and let

$$
\psi:(0,1) \rightarrow \mathbf{R}, \quad \alpha \mapsto-\alpha \theta \ln \alpha-(1-\alpha) \theta \ln (1-\alpha)+r \ln \left(1-\frac{1-(1-\alpha \theta)^{k}}{2^{k}-1}\right)
$$

If there is a number $a \in(0,1)$ such that

$$
\sup _{a<\alpha \leq 1} \psi(\alpha)+\rho / 2^{k}<0
$$

then $\boldsymbol{\Phi}_{t}$ is $2 a \theta$-condensed.

Proof. Let $(\Phi, \sigma)$ be a pair chosen from the planted distribution $\mathcal{P}_{k}(n, m)$. For $\alpha>0$ we let

$$
X_{\alpha}=\left|\left\{\tau \in \mathcal{S}\left(\Phi_{t, \sigma}\right): \operatorname{dist}(\sigma, \tau)=\alpha \theta n\right\}\right| .
$$

Then $\mathrm{E} X_{\alpha} \leq\left(\begin{array}{c}\theta n \\ \alpha \theta n\end{array}\right)\left[1-\frac{1-(1-\alpha \theta)^{k}}{2^{k}-1}\right]^{m}$ and taking logarithms we obtain $\frac{1}{n} \ln \mathrm{E} X_{\alpha} \leq \psi(\alpha)$. Hence, $\frac{1}{n} \ln \mathrm{E} X_{\alpha}<$ $-\rho / 2^{k}$ for $\alpha>a$ by $(38)$. Thus, by Markov's inequality we have

$$
\mathrm{P}\left[\exists \tau \in \mathcal{S}_{t}\left(\Phi_{t, \sigma}\right): d(\sigma, \tau) \geq a \theta n\right] \leq \theta n \cdot \exp \left(-\left(\Omega(1)+\rho / 2^{k}\right) n\right)<\exp \left(-\rho n / 2^{k}\right)
$$

Therefore, the assertion follows from Corollary 4.2

Lemma E.7 Suppose that $\rho \leq k \ln 2-2 \ln k$ and $\theta=\left(1-1 / \rho^{2}\right) \frac{\rho}{k \ln 2}$. Moreover, assume that $\rho \geq \rho_{0}$ and $k \geq k_{0}$ for certain constants $\rho_{0}, k_{0}$. Let $a=\exp (2-\rho)$. Then (38) is satisfied.

Proof. Let $h(\cdot)$ be the entropy function. We have

$$
\psi(\alpha) \leq \theta h(\alpha)-\frac{\rho}{k}(1-\exp (-\alpha k \theta))
$$

To bound the r.h.s., we are going to consider several cases.

Case 1: $\alpha \leq 1 /(k \rho \theta)$. As $\alpha \geq a=\exp (2-\rho)$, we obtain

$$
\psi(\alpha) \leq \alpha \theta[1-\ln \alpha-\rho+\alpha k \rho \theta / 2] \leq \alpha \theta\left[\frac{\alpha k \rho \theta}{2}-1\right] \leq-\alpha \theta / 2 .
$$

The assumption $\rho \leq k \ln 2-2 \ln k$ ensures that the last term is smaller than $-\rho / 2^{k}$.

Case 2: $1 /(k \rho \theta)<\alpha<1 /(k \theta)$. We have

$$
\begin{array}{rlrl}
\psi(\alpha) & \leq \alpha \theta[1-\ln \alpha-\rho+\alpha k \rho \theta / 2] & & \\
& \leq \alpha \theta\left[1+\ln (k \rho \theta)-\rho+\frac{\alpha k \rho \theta}{2}\right] & & \\
& \leq \alpha \theta[1+\ln (k \rho \theta)-\rho / 2] & & {[\operatorname{as} \alpha<1 /(k \theta)]} \\
& \leq \alpha \theta[1-\ln \ln 2+2 \ln \rho-\rho / 2] & & {\left[\operatorname{as} \theta \leq \frac{\rho}{k \ln 2}\right]} \\
& \leq-\alpha \theta \rho / 4 . &
\end{array}
$$

The assumption $\rho \leq k \ln 2-2 \ln k$ ensures that the last term is smaller than $-\rho / 2^{k}$.

Case 3: $1 /(k \theta)<\alpha \leq \alpha_{0}=0.15$. We have

$$
\begin{aligned}
\psi(\alpha) & \leq \theta h(\alpha)-\frac{\rho}{k}(1-\exp (-\alpha k \theta)) \leq \theta h\left(\alpha_{0}\right)-\frac{\rho}{k}(1-1 / \mathrm{e}) \\
& \leq \frac{\rho}{k}\left[\frac{h\left(\alpha_{0}\right)}{\ln 2}-1+1 / \mathrm{e}\right]
\end{aligned}
$$

The choice of $\alpha_{0}$ ensures that the last term is smaller than $-\rho / 2^{k}$. 
Case 4: $\alpha_{0}<\alpha$. As $k \theta=\left(1-1 / \rho^{2}\right) \rho / \ln 2$, we get

$$
\begin{aligned}
\psi(\alpha) & \leq \theta h(\alpha)-\frac{\rho}{k}(1-\exp (-\alpha k \theta)) \leq \theta \ln 2-\frac{\rho}{k}\left(1-\exp \left(-\alpha_{0}\left(1-1 / \rho^{2}\right) \rho / \ln 2\right)\right) \\
& \leq \frac{\rho}{k}\left[\exp \left(-\alpha_{0} \rho\right)-1 / \rho^{2}\right]
\end{aligned}
$$

The last term is smaller than $-\rho / 2^{k}$.

Proof.[Theorem B.2, part 2] Let $\theta_{0}=\left(1-1 / \rho^{2}\right) \rho /(k \ln 2)$ and $t_{0}=\left(1-\theta_{0}\right) n$. Suppose that $\theta \geq \theta_{0}$. Then $\boldsymbol{\Phi}_{t}$ is obtained from $\boldsymbol{\Phi}_{t_{0}}$ by assigning some further variables. Therefore,

$$
\max \left\{d(\sigma, \tau): \sigma, \tau \in \mathcal{S}\left(\boldsymbol{\Phi}_{t}\right)\right\} \leq \max \left\{d(\sigma, \tau): \sigma, \tau \in \mathcal{S}\left(\boldsymbol{\Phi}_{t_{0}}\right)\right\} .
$$

Hence, Proposition E.6 and Lemma E.7 imply that $\boldsymbol{\Phi}_{t}$ is $\exp (2-\rho)$-condensed w.h.p.

\section{E.3 Pairwise distances}

Recall that $\Phi_{t, \sigma}$ denotes the formula obtained by substituting the values $\sigma\left(x_{i}\right)$ for $x_{i}$ for $1 \leq i \leq t$.

Lemma E.8 Suppose that $\theta \geq \frac{\rho}{k \ln 2}\left(1+1 / \rho^{2}+k / 2^{k-2}\right)$. Let $(\Phi, \sigma)$ be a pair chosen from the distribution $\mathcal{U}_{k}(n, m)$. W.h.p. we have

$$
\left|\left\{\tau \in \mathcal{S}\left(\Phi_{t, \sigma}\right): \operatorname{dist}\left(\tau, \sigma_{t}\right) \leq 0.49 \theta n\right\}\right| \leq \exp (-\Omega(n))\left|\mathcal{S}_{t}(\Phi)\right|
$$

Proof. We need to work with the function

$$
\psi(\alpha)=-\alpha \theta \ln \alpha-(1-\alpha) \theta \ln (1-\alpha)+\frac{2^{k} \rho}{k} \ln \left(1-\frac{1-(1-\alpha \theta)^{k}}{2^{k}-1}\right) .
$$

By CorollaryC.1, w.h.p. $\frac{1}{n} \ln \left|\mathcal{S}\left(\Phi_{t}\right)\right| \geq \theta \ln 2+r \ln \left(1-2^{-k}\right)-\rho / 2^{k}$. From now on, we are going to work with the planted model $\mathcal{P}_{k}^{\prime}(n, m)$. We are going to show that

$$
\sup _{\alpha \leq 0.1} \psi(\alpha)-\theta \ln 2-r \ln \left(1-2^{-k}\right)<-\rho / 2^{k-1} .
$$

Then the assertion follows from Corollary C.2. We have

$$
\begin{aligned}
\psi(\alpha)-\theta \ln 2-\frac{2^{k} \rho}{k} \ln \left(1-2^{-k}\right) & =\theta(h(\alpha)-\ln 2)+\frac{2^{k} \rho}{k} \ln \left[1+\frac{(1-\alpha \theta)^{k}-2^{1-k}\left(1-(1-\alpha \theta)^{k}\right)}{2^{k}-1}\right] \\
& \leq \theta(h(\alpha)-\ln 2)+\frac{\rho}{k}(1-\alpha \theta)^{k}+2^{-k} \\
& \leq \theta(h(\alpha)-\ln 2)+\frac{\rho}{k} \exp (-\alpha k \theta)+2^{-k} .
\end{aligned}
$$

The differential of the last expression with respect to $\theta$ is negative, and thus the function is monotonically decreasing in $\theta$. Therefore, it suffices to consider the minimum value $\theta=\rho /(k \ln 2)$. Thus, we obtain

$$
\psi(\alpha)-\theta \ln 2-\frac{2^{k} \rho}{k} \ln \left(1-2^{-k}\right) \leq \frac{\rho}{k}\left(\frac{h(\alpha)}{\ln 2}-1+\exp (-\alpha \rho / \ln 2)\right)+2^{-k} .
$$

We consider a few different cases.

Case 0: $\alpha<\exp (2-\rho)$. Lemma E.3 shows that $\psi(\alpha) \leq 1 /(k \rho)$ and 30] shows that

$$
\theta \ln 2+2^{k} \frac{\rho}{k} \ln \left(1-2^{-k}\right) \geq \theta \ln 2-\rho / k-\rho / 2^{k}
$$

Hence,

$$
\psi(\alpha)-\theta \ln 2-\frac{2^{k} \rho}{k} \ln \left(1-2^{-k}\right) \leq \frac{1}{k \rho}-\theta \ln 2+\frac{\rho}{k}+\rho / 2^{k}
$$

Since we are assuming that $\theta \geq \frac{\rho}{k \ln 2}\left(1+1 / \rho^{2}+k / 2^{k-2}\right)$, the r.h.s. is smaller than $\rho / 2^{k-1}$. 
Case 1: $\exp (2-\rho) \leq \alpha \leq \exp (-\rho / 2)$. Bounding the exponential by a quadratic function, we get

$$
\begin{aligned}
\psi(\alpha)-\theta \ln 2-r \ln \left(1-2^{-k}\right) & \leq \frac{\alpha \rho}{k \ln 2}\left[1-\ln \alpha-\rho+\frac{\alpha \rho^{2}}{4 \ln 2}\right]+2^{-k} \\
& \leq \frac{\alpha \rho}{k \ln 2}\left[-1+\frac{(\alpha \rho)^{2}}{2 \ln 2}\right]+2^{-k}<-\rho / 2^{k-1}
\end{aligned}
$$

provided that $\rho_{0} \leq \rho \leq k \ln 2-2 \ln k$.

Case 2: $\exp (-\rho / 2) \leq \alpha \leq 1 /(2 \rho)$. Bounding the exponential by a quadratic function, we get

$$
\psi(\alpha)-\theta \ln 2-r \ln \left(1-2^{-k}\right) \leq \frac{\alpha \rho}{k \ln 2}\left[1-\ln \alpha-\rho+\frac{(\alpha \rho)^{2}}{2 \ln 2}\right]+2^{-k}<-\rho / 2^{k-1},
$$

provided that $\rho_{0} \leq \rho \leq k \ln 2-2 \ln k$.

Case 3: $1 /(2 \rho)<\alpha \leq 10 \ln (2) / \rho$. Suppose that $\alpha=x \ln (2) / \rho$ for some $1 / 2 \leq x \leq 10 \ln 2$. Then

$$
\psi(\alpha)-\theta \ln 2-r \ln \left(1-2^{-k}\right) \leq \frac{\rho}{k}\left[\frac{x \ln 2}{\rho}(1-\ln x-\ln \ln 2+\ln \rho)-1+\exp (-x)\right]+2^{-k} .
$$

As $x$ remains bounded away from 0 , the term $\exp (-x)-1$ is strictly negative. Thus, the entire expression is smaller than $-\rho / 2^{k-1}$ for $\rho \geq \rho_{0}$ sufficiently large.

Case 4: $10 \ln (2) / \rho<\alpha \leq 0.49$. We have

$$
\psi(\alpha)-\theta \ln 2-r \ln \left(1-2^{-k}\right) \leq \frac{\rho}{k}\left(\frac{h(0.1)}{\ln 2}-1+\exp (-10)\right)+2^{-k} .
$$

The r.h.s. is clearly smaller than $-\rho / 2^{k-1}$.

LemmaE.8 directly implies the third part of Theorem B.2

\section{F Proof of Theorem B.3}

\section{F.1 Bounding the marginals away from 0,1}

Here we prove the first part of Theorem B.3. We may assume that $\theta \geq \rho /(k \ln 2)$. The goal is to show that the marginals of a substantial fraction of the variables $x_{t+1}, \ldots, x_{n}$ are bounded away from 0,1 .

We set up an auxiliary graph $\mathcal{G}$ whose vertices are all pairs $(x, \tau)$ of variables $x \in V_{t}$ and assignments $\tau \in \mathcal{S}\left(\Phi_{t}\right)$. A pair $(x, \tau)$ is connected by an edge with another pair $\left(x, \tau^{\prime}\right)$ if $\tau(x)=\tau^{\prime}(x)$. (Thus, the graph consists of components $(x, \cdot)$ with $x \in V_{t}$.) LemmaE.8 implies the following.

Corollary F.1 Let $\Phi_{t}$ is the formula obtained through the experiment U1-U4. W.h.p. we have $2|E(\mathcal{G})| \leq$ $0.511\left|\mathcal{S}_{t}(\Phi)\right|^{2} \theta n$.

Proof. We count the number of edges from each assignment $\tau$. By Lemma E.8, almost all assignments $\tau$ are such that the 'overlap' with almost all other assignments $\tau^{\prime}$ is at most $0.51 \theta n$. For such assignments, the number of edges incident with $\left\{(\tau, x): x \in V_{t}\right\}$ is bounded by $(1+o(1)) 0.51 \theta n$.

To bound the marginals away from 0,1 , assume that indeed $2|E(\mathcal{G})| \leq 0.511\left|\mathcal{S}_{t}(\Phi)\right|^{2} \theta n$. Any variable $x$ whose marginal does not lie in $(0.01,0.99)$ is such that the set $\left\{(\tau, x): \tau \in \mathcal{S}\left(\Phi_{t}\right)\right\}$ induces at least $(1+$ $o(1)) 0.99 \mathcal{S}\left(\Phi_{t}\right)^{2} / 2$ edges. Hence, if we let $\nu$ be the number of such variables, then $(1+o(1)) 0.99 \mathcal{S}\left(\Phi_{t}\right)^{2} \nu \leq$ $2|E(\mathcal{G})| \leq 0.511\left|\mathcal{S}_{t}(\Phi)\right|^{2} \theta n$. Hence, $\nu \leq \frac{0.511+o(1)}{0.99} \theta n \leq \frac{2}{3} \theta n$. 


\section{F.2 Concentration of the marginals about 0,1}

To prove the second part of Theorem B.3. we need the following lemma.

Lemma F.2 Suppose that $\theta \leq \rho /(k \ln 2)$. Let $(\Phi, \sigma)$ be a pair chosen from the distribution $\mathcal{U}_{k}(n, m)$. W.h.p. there is no set of variables $Z \subset V_{t}$ of size $2 \mathrm{kn} / 2^{k} \leq|Z| \leq(\mathrm{e} \rho)^{-4} \theta n$ such that each variable in $Z$ supports two clauses under $\sigma$, each of which contains an occurrence of a variable in $Z$ that evaluates to 'false' under $\sigma$.

Proof. We work with the planted model $\mathcal{P}_{k}^{\prime}(n, m)$. Let $p$ be such that the expected number of clauses is $m$, i.e., $\left(2^{k}-1\right)\left(\begin{array}{l}n \\ k\end{array}\right) p=m$. Then the probability that a given set $Z$ of size $z$ is 'bad' is bounded by

$$
\left(z\left(\begin{array}{c}
n \\
k-2
\end{array}\right) p\right)^{2 z} \leq(\alpha k \theta \rho)^{2 z}, \text { with } \alpha=z /(\theta n)
$$

Thus, the probability that there is a bad set of size $z$ is bounded by

$$
\left(\begin{array}{c}
\theta n \\
z
\end{array}\right)(\alpha k \theta \rho)^{2 z} \leq\left(\frac{\mathrm{e} \theta n}{z}\right)^{z}(\alpha k \theta \rho)^{2 z}=\left[\mathrm{e} \alpha(k \theta \rho)^{2}\right]^{z} \leq\left(\mathrm{e} \alpha \rho^{4} / \ln ^{2} 2\right)^{z} \leq \exp (-z)
$$

The assumption on $z$ ensures that this is sufficiently small to move from the planted model to $\mathcal{U}_{k}(n, m)$ via Corollary C.2

Proof.[Theorem B.3, part 2] If $k \theta<\ln (\rho) / 2$, then the existence of forced variables immediately implies part 2 of Theorem B.3. Thus, let us assume that $\ln (\rho) / 2 \leq k \theta \leq \rho / \ln 2$. Let $(\Phi, \sigma)$ be a pair chosen from the distribution $\mathcal{U}_{k}(n, m)$. Let $S$ be the set of rigid variables; by Theorem B.1 we have $|S| \geq \rho^{3} \exp (-\rho) \theta n$ w.h.p. Define an auxiliary bipartite graph as follows. The vertices of the graph are the variables in $S$ and the satisfying assignments $\mathcal{S}\left(\Phi_{t, \sigma}\right)$. Each variable $x \in S$ is connected with all $\tau \in \mathcal{S}\left(\Phi_{t, \sigma}\right)$ such that $\tau(x) \neq \sigma(x)$. By the Lemma F. 2 and because $\Phi$ is $\exp (2-\rho)$-condensed (part 2 of Theorem B.2), there is $\varepsilon_{k} \rightarrow 0$ such that the number of edges of this bipartite graph is bounded by $k 2^{1-k} n\left|\mathcal{S}_{t}(\Phi)\right|$. Hence, the degree sum over the variables satisfies

$$
\sum_{x \in S} d(x) \leq k 2^{2-k} n\left|\mathcal{S}_{t}(\Phi)\right|
$$

We may assume without loss of generality that $\sigma=\mathbf{1}$ is the all-true assignment. Then the marginal $\mu(x)$ equals $1-d(x) /\left|\mathcal{S}_{t}(\Phi)\right|$. Hence, the above bound on the degree sum shows that $\mu(x) \leq 2^{-k / 2}$ for all but $0.01 \theta n$ variables $x \in S$.

Proof.[Theorem 2.4 part 4] This follows directly by applying Lemma F.2] to the self-contained set obtained in Appendix D.2

\section{G Belief propagation}

The proof of Theorem 2.7 is based on results from [8]. These results show that, in order to obtain Theorem 2.7 we essentially have to verify that the outcome $\boldsymbol{\Phi}_{t}$ of the experiment $\mathbf{U} \mathbf{1}-\mathbf{U} \mathbf{4}$ enjoys certain quasi-randomness properties. We begin by stating the necessary properties. To this end, we define

$$
\delta_{t}=\exp (-c \theta k)
$$

where $c>0$ is a small absolute constant (independent of $k, r, t, n$ ).

Fix a $k$-CNF $\Phi$ and an assignment $\sigma \in\{0,1\}^{V}$. Let $\Phi_{t, \sigma}$ denote the CNF obtained from $\Phi$ by substituting $\sigma\left(x_{1}\right), \ldots, \sigma\left(x_{k}\right)$ for $x_{1}, \ldots, x_{t}$ and simplifying. Let $G=G\left(\Phi_{t, \mathrm{id}, \mathbf{1}}\right)$ denote the factor graph. For a variable $x \in V_{t}$ and a set $Q \subset V_{t}$ let

$$
N_{\leq 1}(x, Q)=\{b \in N(x):|N(x) \cap Q \backslash\{x\}| \leq 1 \wedge 0.1 \theta k \leq|N(b)| \leq 10 \theta k\} .
$$

Thus, $N_{\leq 1}(x, Q)$ is the set of all clauses that contain $x$ (which may or may not be in $Q$ ) and at most one other variable from $Q$. In addition, there is a condition on the length $|N(b)|$ of the clause $b$ in the decimated formula $\Phi_{t, \sigma}$. Observe that having assigned the first $t$ variables, we should 'expect' the average clause length to be $\theta k$. For a linear map $\Lambda: \mathbf{R}^{V_{t}} \rightarrow \mathbf{R}^{V_{t}}$ let $\|\Lambda\|_{\square}$ signify the norm

$$
\|\Lambda\|_{\square}=\max _{\zeta \in \mathbf{R}^{V_{t} \backslash\{0\}}} \frac{\|\Lambda \zeta\|_{1}}{\|\zeta\|_{\infty}} .
$$


Definition G.1 Let $\delta>0$. We say that $(\Phi, \sigma)$ is $(\delta, t)$-quasirandom if $\Phi$ satisfies $\mathbf{Q 0}$ and $\Phi_{t, \sigma}$ satisfies $\mathbf{Q 1 - Q 4}$ below.

Q0. There are no more than $\ln \ln n$ redundant clauses. Moreover, no variable occurs in more than $\ln n$ clauses of $\Phi$.

Q1. No more than $10^{-5} \delta \theta n$ variables occur in clauses of length less than $\theta k / 10$ or greater than $10 \theta k$. Moreover, there are at most $10^{-4} \delta \theta n$ variables $x \in V_{t}$ such that

$$
(\theta k)^{3} \delta \cdot \sum_{b \in N(x)} 2^{-|N(b)|}>1 .
$$

Q2. If $Q \subset V_{t}$ has size $|Q| \leq \delta \theta n$, then there are no more than $10^{-4} \delta \theta n$ variables $x$ such that either

$$
\begin{aligned}
\sum_{b \in N(x):|N(b) \cap Q \backslash\{x\}|=1} 2^{-|N(b)|} & >\rho(\theta k)^{5} \delta, \text { or } \\
\sum_{b \in N(x):|N(b) \cap Q \backslash\{x\}|>1} 2^{|N(b) \cap Q \backslash\{x\}|-|N(b)|} & >\frac{\delta}{\theta k}, \text { or } \\
\left|\sum_{b \in N_{\leq 1}(x, Q)} \frac{\operatorname{sign}(x, b)}{2^{|N(b)|}}\right| & >\frac{\delta}{1000} .
\end{aligned}
$$

Q3. For any $0.01 \leq z \leq 1$ and any set $Q \subset V_{t}$ of size $0.01 \delta(n-t) \leq|Q| \leq 100 \delta(n-t)$ we have

$$
\sum_{b:|N(b) \cap Q| \geq z|N(b)|}|N(b)| \leq 1.01|Q| / z .
$$

Q4. For any set $Q \subset V_{t}$ of size $|Q| \leq 10 \delta(n-t)$ the linear operator

$$
\Lambda_{Q}: \mathbf{R}^{V_{t}} \rightarrow \mathbf{R}^{V_{t}}, \quad \Gamma \mapsto\left(\sum_{b \in N_{\leq 1}(x, Q)} \sum_{y \in N(b) \backslash\{x\}} 2^{-|N(b)|} \cdot \operatorname{sign}(x, b) \operatorname{sign}(y, b) \Gamma_{y}\right)_{x \in V_{t}}
$$

has norm $\left\|\Lambda_{Q}\right\|_{\square} \leq \delta^{4} \theta n$.

With respect to Q0, we have

Lemma G.2 ([|8]) The random formula $\mathbf{\Phi}$ satisfies condition $\mathbf{Q 0}$ w.h.p., for any density $0<r=m / n \leq 2^{k} \ln 2$.

Let $\Phi$ be a $k$-CNF and let $\delta>0$. For a number $\delta>0$ and an index $l>t$ we say that $x_{l}$ is $(\delta, t)$-biased if the result $\mu_{x_{l}}\left(\Phi_{t, \sigma}, \omega\right)$ of the BP computation on $\Phi_{t, \sigma}$ differs from $\frac{1}{2}$ by more than $\delta$, i.e.,

$$
\left|\mu_{x_{l}}\left(\Phi_{t, \sigma}, \omega\right)-1 / 2\right|>\delta
$$

Moreover, $(\Phi, \sigma)$ is $(\delta, t)$-balanced if no more than $\delta \theta n$ variables are $(\delta, t)$-biased.

Theorem G.3 ([8]) There is $\rho_{0}>0$ such that for any $k, r$ satisfying $\rho_{0} \cdot 2^{k} / k \leq r \leq 2^{k} \ln 2$ and $n$ sufficiently large the following is true. Suppose $(\Phi, \sigma)$ is $\left(\delta_{t}, t\right)$-quasirandom for some $1 \leq t \leq T=\left(1-\ln (\rho) /\left(c^{2} k\right)\right) n$. Then $(\Phi, \sigma)$ is $\left(\delta_{t}, t\right)$-balanced.

At the end of this section, we will verify that random formulas chosen from the distribution $\mathcal{P}_{k}^{\prime}(n, m)$ are indeed quasirandom.

Proposition G.4 There exists a constant $\rho_{0}>0$ such that for any $k, r$ satisfying $\rho_{0} \cdot 2^{k} / k \leq r \leq 2^{k} \ln 2$ there is $\xi=\xi(k, r)>0$ so that for $n$ large and $\delta_{t}$, $T$ as in Theorem $G .3$ the following is true. Let $(\Phi, \sigma)$ be a pair chosen from the planted model $\mathcal{P}_{k}^{\prime}(n, m)$, given that $\sigma=1$ is the all-true assignment. Then

$$
\mathrm{P}\left[(\Phi, \sigma) \text { is }\left(\delta_{t}, t\right) \text {-quasirandom } \mid \mathbf{Q} \mathbf{0}\right] \geq 1-\exp \left[-\rho 2^{1-k} n\right]
$$

for any $1 \leq t \leq T$.

Finally, Theorem 2.7 follows by combining Corollary 4.2 Theorem G.3, and Proposition G.4. 


\section{G.0.1 Proof of Proposition G.4}

Let $\boldsymbol{\Phi}^{\prime}=\boldsymbol{\Phi}_{k}^{\prime}(n, m)$ be a random formula obtained by including each possible clause with probability $p=$ $m /\left(2^{k}\left(\begin{array}{l}n \\ k\end{array}\right)\right)$ independently.

Proposition G.5 ([8, Appendix E]) There exists a constant $\rho_{0}>0$ such that for any $k, r$ satisfying $\rho_{0} \cdot 2^{k} / k \leq$ $r \leq 2^{k} \ln 2$ for $n$ large and $\delta_{t}$, T as in (39) the following properties hold for a random formula $\boldsymbol{\Phi}^{\prime}$ with probability at least $1-\exp \left[-10 \sum_{s \leq t} \delta_{s}\right]$ for any $1 \leq t \leq T$, given that $\boldsymbol{\Phi}^{\prime}$ satisfies $\mathbf{Q} \mathbf{0}$.

1. Q1 and $\mathbf{Q 3}$ are satisfied.

2. For any set $Q$ of size $|Q| \leq \delta \theta n$ there are at most $10^{-5} \delta \theta n$ variables $x$ that satisfy either (41), (42), or

$$
\left|\sum_{b \in N_{\leq 1}(x, Q)} \frac{\operatorname{sign}(x, b)}{2^{|N(b)|}}\right|>\frac{\delta}{2000} .
$$

3. For any $Q$ the operator $\Lambda_{Q}$ from (44) satisfies $\left\|\Lambda_{Q}\right\|_{\square} \leq \delta^{4}(n-t) / 2$

Let $\boldsymbol{\Phi}_{t}$ be the formula obtain from $\boldsymbol{\Phi}$ by substituting the value 'true' for $x_{1}, \ldots, x_{t-1}$ and simplifying. Since the $\delta_{s}$ form a geometric sequence, we have

$$
\Sigma_{t}=\sum_{s \leq t} \delta_{s} \sim \frac{n}{c k \exp (c \theta k)} .
$$

Observe that

$$
\theta \delta n>10^{15} \Sigma_{t}
$$

if $\rho \geq \rho_{0}$ is chosen sufficiently large.

Lemma G.6 There exists a constant $\rho_{0}>0$ such that for any $k, r$ satisfying $\rho_{0} \cdot 2^{k} / k \leq r \leq 2^{k} \ln 2$ the following is true for the random formula $\boldsymbol{\Phi}^{\prime}$ with probability at least $1-\exp \left(-\rho 2^{2-k} n\right)$.

1. The total number of all-negative clauses is bounded by $2^{1-k} m$.

2. For each variable $x \in V_{t}$ let $N_{x}$ be the number of all-negative clauses in which $x$ appears. Then the number of variables $x \in V_{t}$ with $N_{x}>2^{0.01 \theta k}$ is bounded by $\delta^{2} \theta n$.

Proof. The first assertion simply follows from Chernoff bounds. With respect to the second assertion, assume that the first claim occurs, i.e., the total number of all-negative clauses is bounded by $2^{1-k} m=2 \rho n / k$. Then for each variable the average number of occurrences in such clauses is bounded by $2 \rho$. Therefore, the total number of variables that occur more than $2^{0.01 \theta k}$ times is bounded by $2 \rho \cdot 2^{-0.01 \theta k} n$. By symmetry, the number of such variables that are amongst the last $\theta n$ variables is (asymptotically) binomially distribution with mean $2 \rho \cdot 2^{-0.01 \theta k} \theta n$. Therefore, the second assertion follows from Chernoff bounds.

Proof.[Proposition G.4 Let $(\Phi, \sigma)$ be a random pair chosen from the distribution $\mathcal{P}_{k}^{\prime}(n, m)$. We may assume without loss of generality that $\sigma$ is the all-true assignment. Thus, the formula $\Phi$ is obtained by including each clause that does not consist of negative literals only with probability $p=m /\left(\left(2^{k}-1\right)\left(\begin{array}{l}n \\ k\end{array}\right)\right)$ independently. Now, let $\Phi^{\prime}$ be the formula obtained by addition to $\Phi$ each of the $\left(\begin{array}{l}n \\ k\end{array}\right)$ all-negative clauses independently with probability $p$. Then $\Phi^{\prime}$ has distribution $\boldsymbol{\Phi}_{k}^{\prime}\left(n, m \cdot \frac{2^{k}}{2^{k}-1}\right)$. Thus, with probability at least $1-\exp \left[-10 \sum_{s \leq t} \delta_{s}\right]$ the formula $\Phi^{\prime}$ has the properties 1.-3. from Proposition G.5 Let us condition on this event.

Since $\Phi^{\prime}$ contains $\Phi$ as a sub-formula, the fact that $\Phi^{\prime}$ enjoys properties Q1 and Q3 implies that the same is true of $\Phi$. Furthermore, any variable $x$ for which either (41) or (42) is true in $\Phi$ has the same property in $\Phi^{\prime}$ (because the expressions on the left hand side are monotone with respect to the addition of clauses). With respect to the expression in (43), we decompose the sum for the pair $(\Phi, \sigma)$ as

$$
S_{x}(\Phi, \sigma)=S_{x}\left(\Phi^{\prime}, \sigma\right)-R_{x}
$$

where $R_{x}$ sums over all clauses that are in $\Phi^{\prime}$ but not in $\Phi$. Due to Q1, we may assume that only clauses of length at least $0.1 \theta k$ occur in the sum $R_{x}$. Thus, letting $N_{x}$ denote the number of clauses in $\Phi^{\prime} \backslash \Phi$ containing $x$, we get 
$\left|R_{x}\right| \leq 2^{-0.1 \theta k} N_{x}$. The second part of LemmaG.6 implies that for all but $\delta^{2} \theta n$ variables we have $N_{x} \leq 2^{0.01 \theta k}$. Hence, $R_{x}$ is tiny for all but $\delta^{2} \theta n$ variables. This shows that $\Phi$ satisfies $\mathbf{Q 2}$.

With respect to $\mathbf{Q 4}$, let $D$ be the difference of the two linear operators for $\Phi$ and $\Phi^{\prime}$. Only clauses of length at least $0.1 \theta k$ and at most $10 \theta k$ contribute to $D$. Hence, letting $N$ denote the number of all-negative clauses, we have

$$
\|D\|_{\square} \leq 2^{-0.1 \theta k}(10 \theta k)^{2} N .
$$

Since $N \leq 2^{1-k} m=2 \rho n / k$ by LemmaG.6 we thus get

$$
\|D\|_{\square} \leq 200 \theta n(\theta k) 2^{-0.1 \theta k} .
$$

Hence, the third part of Proposition G.5 implies that $\Phi$ satisfies Q4. 